\title{
Towards the Internet of Underground Things: A Systematic Survey
}

\author{
Nasir Saeed, Senior Member, IEEE, Mohamed-Slim Alouini, Fellow, IEEE, Tareq Y. Al-Naffouri, Senior \\ Member, IEEE
}

\begin{abstract}
This paper provides recent advances in the area of Internet of Underground Things (IoUT) with emphasis on enabling communication technologies, networking issues, and localization techniques. IoUT is enabled by underground things (sensors), communication technology, and networking protocols. This new paradigm of IoUT facilitates the integration of sensing and communication in the underground environment for various industries, such as oil and gas, agriculture, seismic mapping, and border monitoring. These applications require to gather relevant information from the deployed underground things. However, the harsh underground propagation environment including sand, rock, and watersheds do not allow the use of single communication technology for information transfer between the surface and the underground things. Therefore, various wireless and wired communication technologies are used for underground communication. The wireless technologies are based on acoustic waves, electromagnetic waves, magnetic induction and visible light communication while the wired technologies use coaxial cable and optical fibers. In this paper, state-of-art communication technologies are surveyed, and the respective networking and localization techniques for IoUT are presented. Moreover, the advances and applications of IoUT are also reported. Also, new research challenges for the design and implementation of IoUT are identified.
\end{abstract}

Index Terms-Internet of Underground Things, communication, networking, localization, survey

\section{INTRODUCTION}

The population of the world will increase by $31 \%$ in 2050 [1], and therefore will require more natural resources and food to survive. In the next three decades with such increase in population, $71 \%$ more resources are required [2]. This everincreasing demand for resources needs novel technologies to improve the underground exploration for natural resources and to produce more crop. The subsurface environment and agricultural lands provide various natural resources, such as earth minerals, fossil fuels, metal ores, groundwater, and food. To efficiently use all of these resources, Internet of Underground Things (IoUT) is an enabling technology which can provide smart oil and gas fields, smart agriculture fields, and smart seismic quality control. However, implementation of IoUT is a challenging task due to the harsh underground environment which requires low power and small size underground sensors, long-range communication technology, efficient networking solutions, and accurate localization techniques.

This work is supported by Office of Sponsored Research (OSR) at King Abdullah University of Science and Technology (KAUST).

The authors are with the Computer Electrical and Mathematical Sciences \& Engineering (CEMSE) Division, KAUST, Thuwal, Makkah Province, Kingdom of Saudi Arabia, 23955-6900.
The above applications and challenges of IoUT lead to active research in this area. The significant difference between the in-air internet of things and IoUT is the communication media where the sensors (underground things) are buried and communicate through the soil. Note that there is also a possibility to place the underground things in an open underground space, such as mines and tunnels. In this case, the network setup is underground, but the communication between the devices takes place through the air and hence terrestrial wireless technologies, such as radio frequency (RF) and visible light (VLC) are used. There are existing review articles on information and communication systems for mines and tunnels. For example, in [3], the authors briefly discuss the information and communications systems for mining IoT. Similarly, the authors in [4] presented a survey on the applications of wireless sensor networks for underground coal and mines. However, communication through the soil is different where the transmission signal suffers from various underground impairments. Hence, in this paper, we collect the literature on communication, networking, and localization for buried smart objects. ${ }^{1}$

Due to the heterogeneous nature of the soil which consists of sand, rock, and watersheds, communication through it is more challenging. In the past, various solutions were used for underground communications. For example, the use of mud pulse telemetry (MPT) communication systems for oil and gas monitoring dates back to the mid of the $19^{\text {th }}$ century [6]. The MPT systems work on the concept of mud circulation in the pipes for data transmission [7]. Although MPT systems are well-developed for down-hole monitoring, their data rate is low, i.e., in bits per second. To improve the data rates for underground communications, wired cables, such as coaxial cables and fiber optics are also used. These wired solutions provide high data rates, timely, reliable and accurate solutions, especially for the deep underground monitoring. Therefore, wired technologies including coaxial cable and optical fiber are used in many works, such as [8]-[10] for down-hole monitoring. Although wired solutions have the above advantages, they have high complexity and are not scalable solutions. Therefore, wireless solutions are investigated to provide low complex, high data rate, and scalable solutions.

These wireless solutions include acoustic waves, electromagnetic waves (EM), magnetic-induction (MI), and VLC. Acoustic waves are used for the detection of objects un-

${ }^{1} \mathrm{IoT}$ in mining is beyond the scope of this survey since there are existing review articles on this subject, such as [3]-[5]. 
TABLE I: Comparison of underground communication technologies for IoUT.

\begin{tabular}{|c|c|c|c|c|c|c|}
\hline Parameters & EM & Acoustic & Mud pulse & MI & Wired & VLC \\
\hline $\begin{array}{l}\text { Transmission } \\
\text { Range }\end{array}$ & few meters & $\begin{array}{l}\text { In hundred of me- } \\
\text { ters }\end{array}$ & $\begin{array}{l}\text { In hundred of me- } \\
\text { ters }\end{array}$ & In tens of meters & $\begin{array}{l}\text { In hundred of me- } \\
\text { ters }\end{array}$ & In tens of meters \\
\hline Attenuation & High & High & Medium & Low & Low & High \\
\hline Interference & High & Medium & Medium & Low & Low & Low \\
\hline $\begin{array}{l}\text { Installation } \\
\text { cost }\end{array}$ & Medium & Medium & Low & Medium & Low & Low \\
\hline Data rate & In tens of bps & In tens of bps & In tens of bps & In $\mathrm{kbps}$ & In Mbps & In kbps \\
\hline Applications & $\begin{array}{l}\text { Agriculture, seismic } \\
\text { exploration, and } \\
\text { down-hole telemetry }\end{array}$ & $\begin{array}{l}\text { Seismic } \\
\text { exploration, } \\
\text { buried pipeline } \\
\text { monitoring, } \\
\text { and down-hole } \\
\text { telemetry }\end{array}$ & $\begin{array}{l}\text { Down-hole } \\
\text { telemetry }\end{array}$ & $\begin{array}{l}\text { Down-hole } \\
\text { telemetry }\end{array}$ & $\begin{array}{l}\text { Down-hole } \\
\text { telemetry and } \\
\text { buried pipeline } \\
\text { monitoring }\end{array}$ & $\begin{array}{l}\text { Down-hole } \\
\text { telemetry }\end{array}$ \\
\hline Advantages & $\begin{array}{l}\text { High data rate com- } \\
\text { pare to acoustic and } \\
\text { MPT systems, easy } \\
\text { to install, and can } \\
\text { be implemented in } \\
\text { multi-hop fashion }\end{array}$ & $\begin{array}{l}\text { Long transmission } \\
\text { range and high } \\
\text { data rate than } \\
\text { MPT systems }\end{array}$ & $\begin{array}{l}\text { Long communica- } \\
\text { tion range and low } \\
\text { installation cost }\end{array}$ & $\begin{array}{l}\text { High data rate, } \\
\text { support multi-hop } \\
\text { communication, } \\
\text { low interference, } \\
\text { and low } \\
\text { attenuation }\end{array}$ & $\begin{array}{l}\text { High data rate, re- } \\
\text { liable, low latency, } \\
\text { and accurate }\end{array}$ & $\begin{array}{l}\text { Low installation } \\
\text { cost and low } \\
\text { interference }\end{array}$ \\
\hline Disadvantages & $\begin{array}{l}\text { High attenuation and } \\
\text { interference, limited } \\
\text { transmission range, } \\
\text { and require large } \\
\text { antennas }\end{array}$ & $\begin{array}{l}\text { High attenuation } \\
\text { and low data rate } \\
\text { than EM, MI, } \\
\text { wired, and VLC } \\
\text { solutions }\end{array}$ & $\begin{array}{l}\text { Low data rate and } \\
\text { high complexity }\end{array}$ & $\begin{array}{l}\text { Limited } \\
\text { transmission } \\
\text { range, need dense } \\
\text { deployment, } \\
\text { and orientation } \\
\text { between the coils } \\
\text { is required }\end{array}$ & $\begin{array}{l}\text { High complexity } \\
\text { and non-scalable }\end{array}$ & $\begin{array}{l}\text { High attenuation, } \\
\text { require line of } \\
\text { sight, and can } \\
\text { operate only if } \\
\text { the underground } \\
\text { medium is air }\end{array}$ \\
\hline
\end{tabular}

derground, soil moisture detection, and down-hole communications. For example, in [11], the authors used 2 to 6 $\mathrm{kHz}$ frequencies to detect an underground object. Similarly, a frequency of $900 \mathrm{~Hz}$ was used in [12] to find the soil moisture. Recently, the authors in [13] and [14] proposed acoustic waves based wireless data transmission system (SoilComm) for IoUTs that was able to transmit the sensing data over 30 $\mathrm{m}$ distance through the soil. Acoustic waves-based solutions are good for detection purposes, such as underground object detection and soil moisture detection. However, they provide low data rate communication, i.e., in tens of bits per second for down-hole monitoring and also suffers from the acoustic noise and attenuation of the acoustic signal along the drill-pipe [15].

To investigate the use of EM waves for underground communications, various frequencies of the EM spectrum were examined in the past starting from below $500 \mathrm{kHz}$ [16][18] to $120 \mathrm{THz}$ [19]. Depending on the frequency of EM waves, the transmission range of EM signal varies from a few meters (few hundred $\mathrm{kHz}$ ) to centimeters $(\mathrm{THz}$ ). Due to their low penetration depth in the soil, EM waves are mainly used for agriculture applications. For example, in [20] the authors presented EM waves-based IoUT solution for precision agriculture. The EM waves suffer from severe path loss in soil, and therefore their transmission range is low. Especially underground watersheds have a notoriously bad impact on the propagation of EM waves by limiting its transmission range. Alternatively, multi-hop MI-based underground communications is examined in the recent past [21]. Although the MI channel is more robust to the underground environment, it suffers from low transmission range and requires perfect orientation of the transmitter and receiver coils which might be challenging in the underground environment. The research on developing MI-based IoUT is still in the academic phase and faces various challenges. Nevertheless, VLC is also being investigated for the underground communications in gas reservoirs [22]. However, in the VLC based system, the propagation of light is affected by the gas as well as it requires perfect alignment between the LEDs and the photo-detector. In short, among all these solutions, MPT systems, coaxial cable, fiber optics are the commercially available technologies for downhole monitoring while acoustic, EM, MI, and VLC based systems are still in the academic phase of research and are only tested by lab experiments. Table I compares various communication technologies for IoUT.

\section{A. Related Surveys}

There are quite few survey articles published that cover various issues of IoUTs. For example, the work in [20] presents the EM waves based IoUTs for precise agriculture. Moreover, [20] also reviews the academic testbeds and commercial solutions for precise agriculture. In [23], the authors give an overview of MI-based underground wireless sensor networks and present challenges and applications. The authors in [24] present the recent advances and challenges for wireless sensor networks in the oil and gas industry.

The contributions of this article relative to the existing literature on IoUT is summarized as follows:

- Compared to existing papers for IoUTs, this paper provides a deeper understanding of the all relevant communication technologies, networking solutions, and localization techniques which can be used to implement various IoUT-based applications.

- The existing surveys only present the literature on EM and MI-based underground wireless communication networks. However, we also collect the research on acoustic, 


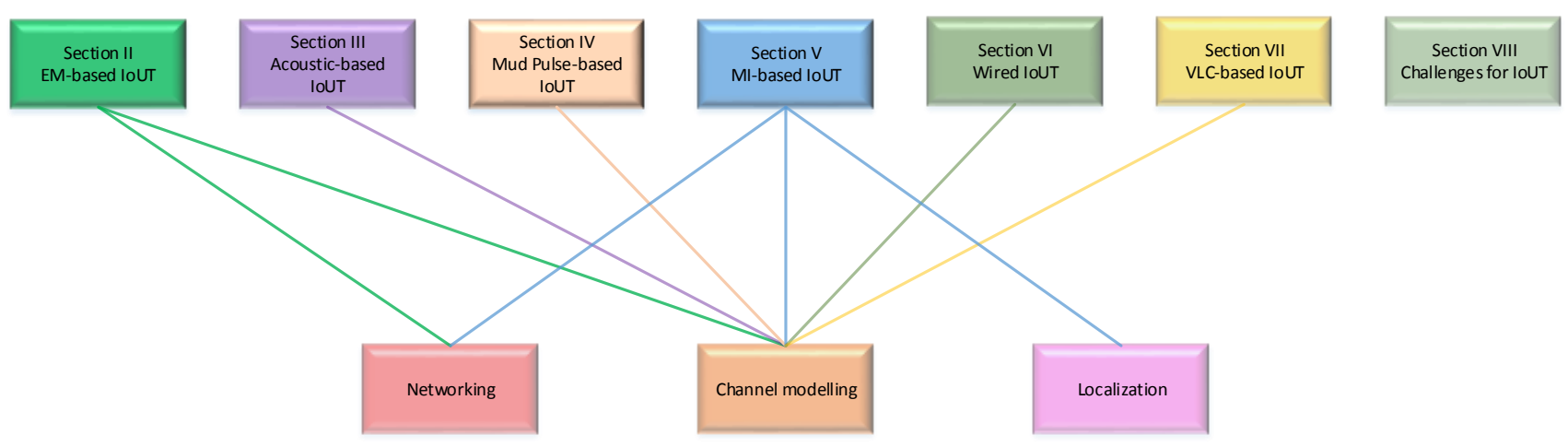

Fig. 1: Organization of the survey.

mud-pulse telemetry, visible light, and wired-based communication technologies for various IoUT applications.

- We survey the key challenges to implement IoUT and explore the relationship between IoUT, big data analytics, cloud, and fog computing.

\section{B. Survey Organization}

The main focus of the paper is to review channel modeling, networking, and localization methods for the current sensing and communication technologies used in IoUT. However, for some of the techniques, such as acoustic, visible light communications, and mud-pulse telemetry, the networking, and localization problem is still an open research problem. Fig. 1 illustrates the organization of this survey. In Section II, we present the literature on channel modeling and networking for EM-based underground communication. Sections III and IV cover the channel model for acoustic and mud pulse telemetry-based IoUTs, respectively. In Section V, channel modeling, networking, and localization for MI-based IoUTs are presented. VLC and wired based solutions are presented in section VI and VII, respectively. Section VIII discusses the advances and challenges of IoUTs in terms of each communication technology. Finally, section IX summarizes and concludes the survey.

\section{EM WAVES FOR IOUT}

EM waves are widely used for underground communication and sensing to enable various applications, such as smart agriculture [25]-[28], smart seismic exploration [29], smart drilling, and smart oil and gas fields [30]-[32]. In this section, we cover the literature on the channel modeling and networking for EM-based IoUTs.

\section{A. Channel Modeling}

Channel modelling for EM-based underground communications dates back to the early 70 's of the $20^{\text {th }}$ century. James et. al investigated the propagation of EM signals through the earth surface where the frequency range of 1 to $10 \mathrm{MHz}$ was experimentally tested in different types of soil [33]. Their results suggest that at low frequencies of the EM spectrum, earth refractive index is large which simplifies the analysis. However, low frequencies suffer from limited bandwidth and low resolution for time of arrival pulses. Therefore, higher frequencies of the EM spectrum were tested in [33] which suffers from high attenuation but have a low dispersion. The authors in [34] further examined the impact of different layers of the soil on the propagation of EM signals where Fast Fourier Transform was used to find the reflection of the incident signals from a three-layered medium. Furthermore, Lytle et. al measured the electrical characteristics of the earth medium for the propagation of underground EM signals [35]. The electrical characteristics of the earth, such as conductivity and dielectric constant have a deceitful effect on the propagation of EM signals. The values of conductivity and dielectric constant significantly influence the selection of antenna, size of the ground screen, transmission loss, phase shift, dispersion, and antenna efficiency. Therefore, conductivity and dielectric constant for various types of soils and rocks were experimentally measured in [35]. The authors in [36] experimentally measured the conductivity of the earth surface at EM frequencies of 3 to $50 \mathrm{MHz}$ at Yosemite national park. William et. al measured high-frequency electromagnetic radiations in the borehole by neglecting the reflections and refractions from the subsurface [37]. The path loss model defined in [37] is given as

$$
\frac{P_{r}}{P_{t}}=\frac{f(\theta) G_{t} A_{r} \exp ^{-2 \alpha d}}{4 \pi r^{2}},
$$

where $P_{r}$ and $P_{t}$ are the received and transmitted power, respectively, $f(\theta)$ is the effective elevation radiation pattern, $G_{t}$ is the gain of transmitting antenna, $A_{r}$ is the effective area of receiving antenna, $\alpha$ is the attenuation factor, and $d$ is the distance between the transmitting and the receiving antenna. In [38], the authors examined EM waves for borehole communications where a data rate of $1 \mathrm{bps}$ was achieved for average conductivity and without using the repeaters while with the use of repeaters, the data rate can reach up to 100 bps. In [39], the authors have shown the impact of soil properties, water content, network topology, and antenna type for the EM waves in the frequency range of 1 to 3 $\mathrm{MHz}$ for underground wireless sensor networks (UGWSNs). It was shown numerically in [39] that the path loss and 
attenuation increases with increase in the humidity and the operating frequency. Consequently, in [40], experiments were conducted in subsoil and topsoil at $300-500 \mathrm{MHz}$ frequencies for buried sensors. Furthermore, attenuation of EM signal for measurement while drilling (MWD) telemetry system was investigated in [41] where the maximum transmission range of 15,000 feet was achieved without using repeaters. Based on the Friis free space path loss model, the authors in [42] provided the formula for the received power in the soil medium as

$$
P_{r}(\mathrm{~dB})=P_{t}(\mathrm{~dB})+G_{t}(\mathrm{~dB})+G_{r}(\mathrm{~dB})-L_{s}(\mathrm{~dB}),
$$

where $P_{t}$ represents the transmit power, $G_{t}$ and $G_{r}$ are the transmit and receive antenna gains, respectively, and $L_{s}=L_{f}+L_{u}$ is the path loss in soil medium. $L_{f}$ and $L_{u}$ are the free space and underground path loss, respectively. Underground path loss $L_{u}$ is calculated by considering the EM waves propagation characteristics in soil, such as operating wavelength and frequency, scattering, and delay distortion. Hence, $L_{u}=L_{\alpha}+L_{\beta}$, where $L_{\alpha}$ and $L_{\beta}$ are attenuations due to transmission loss and wavelength difference of EM signal in soil compared to air, respectively. Therefore, $L_{s}$ is represented in $d B$ as follows

$$
L_{s}=6.4+20 \log (d)+8.69 \alpha d+20 \log (\beta),
$$

where $d$ is the Euclidean distance, $\alpha$ is the attenuation constant, and $\beta$ is the phase shift constant. Both the attenuation and phase shift constants depend on the dielectric properties of the soil. The dielectric properties of the soil are calculated by using the Peplinski principle as follows [43]:

$$
\epsilon_{s}=\epsilon_{r}-j \epsilon_{i}
$$

where $\epsilon_{s}$ is the complex dielectric constant of soil and water mixture consisting of a real part $\epsilon_{r}$ and an imaginary part $\epsilon_{i}$, respectively. The real part of $\epsilon_{s}$ is given as

$$
\epsilon_{r}=1.15\left(1+\frac{\rho_{b}\left(\epsilon_{x}^{\bar{\alpha}}\right)}{\rho_{x}}+m_{v}^{\bar{\beta}} \epsilon_{f}^{\bar{\alpha}}-m_{v}\right)^{\frac{1}{\bar{\alpha}}}-0.68,
$$

where $\rho_{b}$ is the bulk density, $\rho_{s}=2.66$ is density of solid soil, $\bar{\alpha}=0.65, m_{v}$ is the water volume fraction, and $\bar{\beta}=1.2748-$ $0.519 S-0.152 C$ is the empirically determined constants for soil type. The terms $S$ and $C$ represents mass fractions of sand and clay, respectively and their values lies between 0 and 1 . The effective conductivity $\epsilon_{f}$ in (5) is given as

$$
\epsilon_{f}=\frac{\epsilon_{0}-\epsilon_{\infty}}{1+(2 \pi f \tau)^{2}}+\epsilon_{\infty},
$$

where $\epsilon_{0}=80.1$ is the static dielectric constant, $\epsilon_{\infty}=4.9$ is the high frequency limit, $\tau$ is the relaxation time of water, and $f$ is the operating frequency [44]. Similarly the imaginary part $\epsilon_{i}=\left(m_{v}^{\widehat{\beta}} \epsilon_{f}^{\bar{\alpha}}\right)^{\frac{1}{\alpha}}$, where $\tilde{\beta}=1.33797-0.603 S-0.166 C$. Consequently, the attenuation constant $\alpha$ is given as

$$
\alpha=2 \pi f\left(\frac{\mu \epsilon_{r}}{2}\left(\sqrt{1+\left(\frac{\epsilon_{i}}{\epsilon_{r}}\right)^{2}}-1\right)\right),
$$

where $\mu$ is the magnetic permeability. Similarly, the phase shift constant $\beta$ is found as

$$
\beta=2 \pi f\left(\frac{\mu \epsilon_{r}}{2}\left(\sqrt{1+\left(\frac{\epsilon_{i}}{\epsilon_{r}}\right)^{2}}+1\right)\right) .
$$

It is clear from the expression of both the attenuation and phase shift constants that the propagation loss of EM depends on the operating frequency, soil composition, water content, and bulk density. Furthermore, the authors in [42] have investigated the path loss in the presence of two paths between the transmitter and the receiver. The authors have neglected the second path effect in high depth scenarios due to no reflection from the ground surface while for low depth scenario two-path model was considered which is given in $d B$ as follows:

$$
L_{t}=L_{s}-L_{v},
$$

where $L_{v}$ correspond to the second path loss, given as

$$
\begin{aligned}
L_{v}^{2}= & 1+\left(\gamma \exp ^{(-\alpha \Delta(r))^{2}}\right)-2 \gamma \exp ^{(-\alpha \Delta(r))} \\
& \cos \left(\pi-\left(\phi-\frac{2 \pi \Delta(r)}{\lambda}\right)\right),
\end{aligned}
$$

where $\gamma$ and $\phi$ are the amplitude and phase reflection coefficients, respectively, $\lambda$ is the wavelength, and $\Delta(r)=r-d$ is the difference between the two paths. Based on the above channel model, the authors in [45] proposed a testbed for UGWSNs. The authors in [46] also compared the theoretical and measured results for UGWSNs where the above analytical model fits well within $3.45 \mathrm{dBm}$ of the measured data. In [19] EM waves in Terahertz band $(0.1-120 \mathrm{THz})$ were investigated for oil reservoirs. The path loss (in $\mathrm{dB}$ ) for EM signals in the Terahertz band is given in [19] as follows

$$
L_{t o t}=L_{s p}+L_{w}+L_{o},
$$

where $L_{s p}$ is the spreading loss, $L_{w}=k_{w}(f) d$ is the absorption loss due to water, and $L_{o}=k_{o}(f) d$ is the absorption loss due to oil. The spreading loss $L_{s p}=\left(\frac{4 \pi f d}{c}\right)^{2}$, where $d$ is the distance, $f$ is the operating frequency, $c$ is the speed of light, and $k_{w}(f), k_{o}(f)$ are the absorption coefficients of water and oil, respectively. Although the $\mathrm{THz}$ band provides high capacity for UGWSNs, their range is limited to few centimeters. Hence, the concept of low frequency (below 500 $\mathrm{kHz}$ ) was introduced in [16]-[18] to achieve more considerable transmission distance (in tens of meters) for UGWSNs. The received power formula for the EM signals in [16]-[18] is given as

$$
P_{r}=\kappa \frac{\exp ^{-2 \alpha d}}{d^{2}}
$$

where $\kappa=\frac{A_{r} \cos \theta}{2 \eta}\left(\frac{I \mu_{0} \omega}{4 \pi}\right)^{2}, \theta$ is the phase angle, $\eta$ is the intrinsic wave impedance, $\mu_{0}$ is the permeability of air, $\omega$ is the angular frequency, and $I$ is the current.

The impact of the carrier frequency, transmission distance, depth, and modulation type was experimentally tested in [47][49] for UGWSNs. The optimum frequency range in 10$100 \mathrm{MHz}$ was identified in [50] for energy harvesting in UGWSNs. To reduce the battery consumption and to improve the signal to noise ratio (SNR) at the receiver, a code division 
multiplexing scheme was proposed in [51]. Consequently, the impact of soil type on multi-carrier modulation was examined in [52] which showed that the data rate of $124 \mathrm{Mbps}$ is achievable for the transmission distance of $12 \mathrm{~m}$ for IoUT. In [53], the authors used pulse amplitude modulation, quadrature phase shift keying, m-ary quadrature amplitude modulation, and Gaussian minimum shift keying for IoUT. Moreover, it was shown in [53] that adaptive equalization improve the performance of the underground channel. Furthermore, the authors in [54] tested 97-130 MHz EM frequencies for underground radio propagation by using the same model in [42]; however, the error was almost $50 \%$ at such high frequencies. In [55], a real-time soil moisture sensing and permittivity estimation system called Di-Sense was proposed to implement IoUT for agricultural applications. Software defined radiobased experiments were conducted where the permittivity and soil moisture were calculated at a depth of $4 \mathrm{~cm}$ and horizontal distance of 1 to $15 \mathrm{~m}$, for the frequency range of 100-500 MHz.

Recently, the authors in [56] investigated the soil effects, the orientation of the buried antenna, and depth on the underground to the above ground wireless communication link. The empirical path loss for underground to above ground link is given in logrithmic scale as follows [56]

$$
L_{a g}=-147.6+20 \log d_{a g}+20 \log f
$$

where $d_{a g}$ is the path length. Consequently, empirical studies were conducted in [57] by using the above model to show the propagation characteristics of the underground to above ground communication link at $2.4 \mathrm{GHz}$ and $433 \mathrm{MHz}$, respectively. The emperical model in [42] and its variants are mostly employed in the literature for EM-based underground communication. However, these emperical models ignore the physical characteristics of subsurface EM field analysis. Therefore, recently in [58], the author proposed an analytical solution for EM-based underground communications which rely on the Maxwell-Poynting theory. The derived expressions in [58] relate the soil properties to the electromagnetic field of the buried dipole antenna. Table II summarizes the literature on channel modeling for EM-based IoUT.

\section{B. Networking}

The literature on channel model for EM-based IoUT is rich. However, few works exist on the routing protocols. In this section, we cover the existing research on networking layer protocols for EM-based IoUT. Fig. 2 and 3 show examples of a multi-hop network for an EM-based oil and gas IoUT and agricultural IoUT, respectively. Due to the limited transmission range of EM waves in the harsh underground environment multi-hop communication is well-suited. For example, the idea of dense sensor networks with multi-hop communication for oil and gas exploration was presented in [29]. Moreover, the authors in [59] developed a TCP/IP based simulator for the IoUT. Furthermore, they evaluated the performance of various multiple access schemes in [60]. The path connectivity problem for EM-based IoUT was investigated in [61] which showed that low volumetric water content and low operating

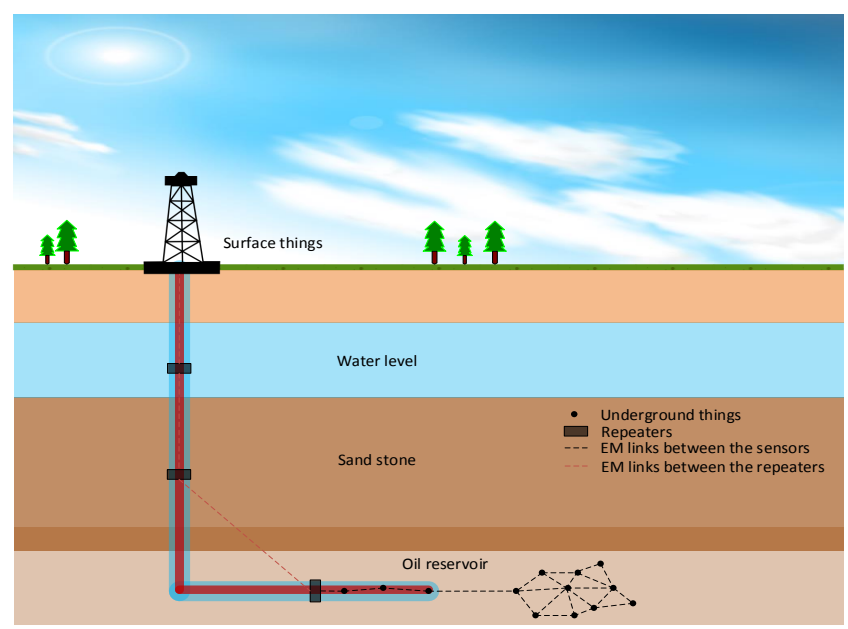

Fig. 2: Network model for EM-based IoUT for oil and gas reservoirs.

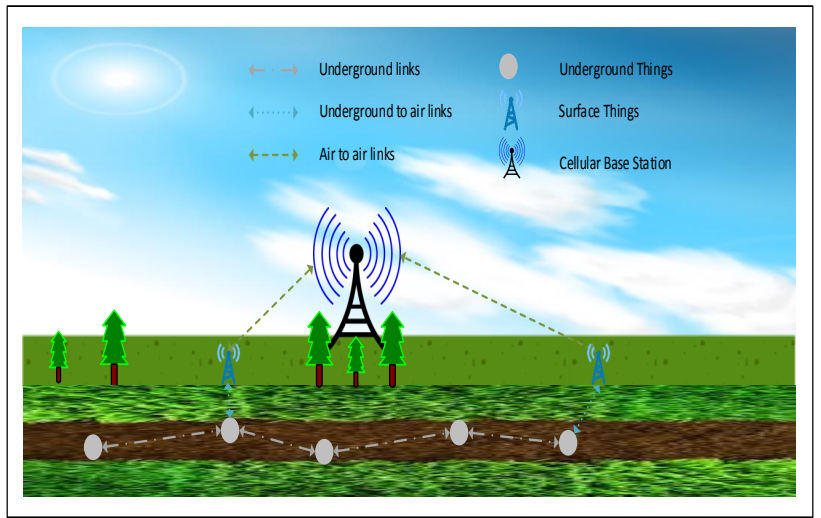

Fig. 3: Network model for EM-based IoUT for agricultural applications.

frequency lead to a higher probability of connectivity. In [62], the throughput of EM-based IoUT was optimized to achieve the QoS requirement. Recently, the influence of soil texture, particle density, and bulk density on the hop count was examined for IoUT where the number of hops between a source and a sink increases with an increase in the water content and clay in the soil. A relay based approach with physical constraints on the relay location, propagation environment, and load balancing was examined in [63] to improve the lifetime of IoUT.

\section{Acoustic WAVES FOR IoUT}

Most of the communication and detection techniques for underground measurements are based on acoustic waves. Geologists use acoustic waves to look for underground resources, such as oil and gas. Acoustic waves are transmitted into the ground, and the reflection is measured from the propagation of the acoustic waves. Moreover, acoustic waves are used in drilling to communicate with underground equipment. Major 
TABLE II: Summary of channel modeling for EM-based IoUT.

\begin{tabular}{|c|c|c|c|c|c|}
\hline Ref. & Data rate & Frequency range & Issue addressed & Applications & Year \\
\hline$[33]$ & - & $1-10 \mathrm{MHz}$ & $\begin{array}{ll}\text { Propagation } & \text { characteris- } \\
\text { tics } & \end{array}$ & Seismic/Agriculture & 1971 \\
\hline [34] & - & - & $\begin{array}{l}\text { Structure of soil effect on } \\
\text { EM waves propagation }\end{array}$ & Seismic/Agriculture & 1973 \\
\hline [35] and [36] & - & 3-50 MHz & $\begin{array}{l}\text { Electrical characteristics } \\
\text { of soil }\end{array}$ & Seismic/Agriculture & 1974 and 1976 \\
\hline [38] & $1-100 \mathrm{bps}$ & - & $\begin{array}{l}\text { EM waves for borehole } \\
\text { communications }\end{array}$ & Oil and Gas & 1990 \\
\hline [39] & - & $1-3 \mathrm{MHz}$ & $\begin{array}{l}\text { Impact of soil and net- } \\
\text { work parameters }\end{array}$ & Agriculture & 1990 \\
\hline [41] & - & $2-6 \mathrm{~Hz}$ & $\begin{array}{l}\text { Investigation of depth on } \\
\text { the signal strength }\end{array}$ & Oil and Gas & 2009 \\
\hline [40] & - & $300-500 \mathrm{MHz}$ & Impact of soil type & Agriculture & 2009 \\
\hline [42] & - & - & $\begin{array}{l}\text { development of the path } \\
\text { loss model }\end{array}$ & Agriculture & 2010 \\
\hline [45] & - & - & Test-bed & Agriculture & 2010 \\
\hline [46] & - & $0.3-1.3 \mathrm{GHz}$ & $\begin{array}{l}\text { Comparison of theoretical } \\
\text { and experimental results }\end{array}$ & Agriculture & 2011 \\
\hline [19] & - & $0.1-120 \mathrm{THz}$ & Channel model & Oil and Gas & 2012 \\
\hline$[16]-[18]$ & - & below $500 \mathrm{kHz}$ & $\begin{array}{l}\text { Propagation characteris- } \\
\text { tics }\end{array}$ & Agriculture & 2012 \\
\hline [50] & - & $10-100 \mathrm{MHz}$ & Energy harvesting & Seismic/Agriculture & 2012 \\
\hline$[47]-[49]$ & - & $433 \mathrm{MHz}$ & $\begin{array}{l}\text { Propagation characteris- } \\
\text { tics }\end{array}$ & Agriculture & 2014-2016 \\
\hline [51] & - & - & $\begin{array}{l}\text { New transmitter and re- } \\
\text { ceiver configurations to } \\
\text { improve the sensor node } \\
\text { lifetime }\end{array}$ & Seismic & 2016 \\
\hline [56] & - & $3.1-10.6 \mathrm{GHz}$ & $\begin{array}{l}\text { Impact of soil on ultra- } \\
\text { wideband underground to } \\
\text { above ground communica- } \\
\text { tion link }\end{array}$ & Agriculture & 2017 \\
\hline [52] & $124 \mathrm{Mbps}$ & $433 \mathrm{MHz}$ & $\begin{array}{l}\text { Multi-carrier modulation } \\
\text { for EM-based IoUT }\end{array}$ & Agriculture & 2017 \\
\hline [53] & - & $100-300 \mathrm{MHz}$ & $\begin{array}{l}\text { Using of the direct, re- } \\
\text { flected, and lateral compo- } \\
\text { nents of the underground } \\
\text { channel to improve the } \\
\text { BER }\end{array}$ & Agriculture & 2017 \\
\hline [57] & - & $\begin{array}{l}433 \mathrm{MHz} \text { and } 2.4 \\
\mathrm{GHz}\end{array}$ & $\begin{array}{l}\text { Influence of depth on the } \\
\text { propagation of EM signal }\end{array}$ & Agriculture & 2017 \\
\hline [54] & - & $97-130 \mathrm{MHz}$ & Soil moisture sensing & Agriculture & 2018 \\
\hline [55] & - & $433 \mathrm{MHz}$ & $\begin{array}{l}\text { Estimation of relative per- } \\
\text { mittivity and soil moisture }\end{array}$ & Agriculture & 2019 \\
\hline [58] & - & - & $\begin{array}{l}\text { Underground channel } \\
\text { modeling by using } \\
\text { Maxwell-Poynting theory }\end{array}$ & Agriculture & 2019 \\
\hline
\end{tabular}

applications of acoustic waves-based IoUTs include smart seismic exploration, earthquake monitoring, buried pipeline monitoring, and smart drilling for oil and gas reservoirs. The research work on acoustic-based underground communications systems is rich and can support many other applications. Based on the signal generation, the acoustic-based methods can be broadly classified into passive and active type methods. In passive acoustic-based methods, the subsurface environment generates an acoustic signal caused by natural events, such as earthquakes, nuclear explosion, and volcanic explosions. In such circumstances, the sensors are placed in the vicinity of the event area. These sensors detect the infrasonic signals which help in the prediction of a natural disaster. Moreover, sudden changes underground, such as rock crack formation, structural transformation, and pipeline leakage can also be detected by using passive acoustic methods. In active acoustic-based methods, the signal is generated by an artificial explosion or vibration which is sent underground to estimate the properties of the earth's subsurface (see Fig. 4). The popular application of such method is reflection-based seismology. Due to the low propagation speed of acoustic waves, they are mostly used for detection purposes in soil rather than for communication. In [11], the authors investigated the speed of sound in the soil. Acoustic signals were transmitted through different samples of soil and received by the hydrophones. The attenuation coefficients were calculated for the frequencies range from 2 to $6 \mathrm{kHz}$. The proposed empirical solution was able to detect an object buried at $40 \mathrm{~cm}$. Similarly, in [64] and [65], soil moisture was measured by using the speed-moisture curves for underground acoustic signals transmission. Moreover, acoustic waves with a frequency of $900 \mathrm{~Hz}$ was used in [12] to estimate the moisture content of the soil. A universal soil loss equation was derived in [66] for acoustic waves propagation in soil at $16 \mathrm{kHz}$ frequency. The soil loss factor due to the erosion is expressed in [66] as $L_{e_{s}}=\rho e_{s} \tau c_{m} \varrho$, where $\rho$ is the soil loss due to rain factor, $e_{s}$ is the soil erosion factor, $\tau$ is the 


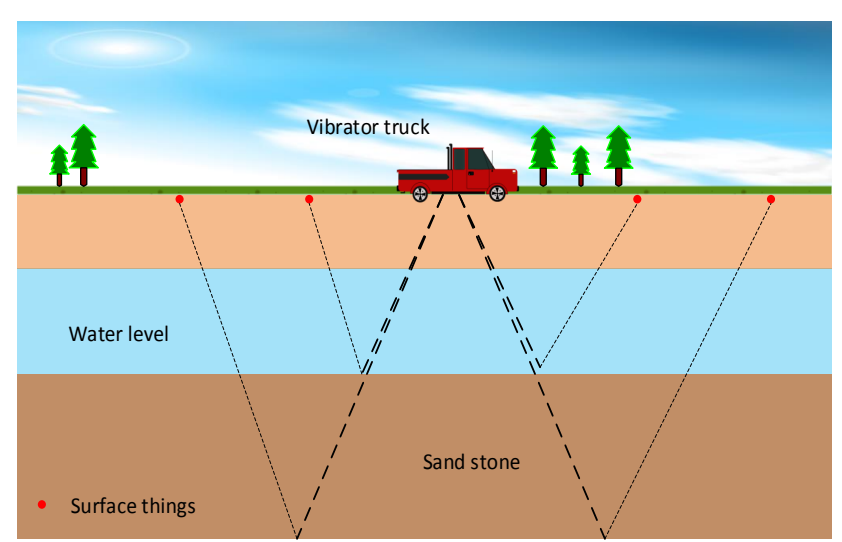

Fig. 4: Architecture of an active acoustic-based IoUT for seismic monitoring.

topographic factor of soil, $c_{m}$ is the cover management, and $\varrho$ is the support practices factor. Recently, the authors in [13] and [14] proposed an acoustic based wireless data transmission system (SoilComm) for IoUTs. SoilComm system was able to transmit the sensing data over $30 \mathrm{~m}$ distance through soil.

In addition to the investigation of soil properties, acoustic waves are widely used for down-hole telemetry purposes. In acoustic telemetry, the steel walls of the drill-string are used as a source of a communication channel. Acoustic-based telemetry system consists of a piezoelectric-electric transmitter underground, repeater at 500-2000 m apart, and a transceiver at the ground surface. The down-hole transmitter encode the sensor's data and convert it into the acoustic signal which then propagates to the surface by the drill string. The drill string consists of pass-bands and stop-bands in the channel. It was found in [15] that attenuation on the drill-string is in the range of 4 to $7 \mathrm{~dB}$ per 1000 feet. The expression for the capacity of the acoustic telemetry system was calculated in [15] as follows

$$
C_{a}=\int \log _{2}\left(1+\frac{P_{s}(f)}{P_{N_{d}}(f)+|H(f)|^{-2} P_{N_{s}}(f)}\right) d f
$$

where $P_{s}, P_{N_{s}}(f)$ and $P_{N_{d}}(f)$ are the power spectra of the signals, surface noise and drilling noise, respectively. The transfer function for the acoustic channel is represented by $H(f)$. Acoustic waves passing through the drill string are highly attenuated and therefore require a sufficient number of repeaters. Additionally, the drilling noise also affects the propagation of acoustic waves along the string. The authors in [15] were able to achieve the data rate of $20 \mathrm{bps}$ at a depth of 3695 feet. In [67], field tests were performed by using acoustic telemetry where data rates of 20,40 , and $60 \mathrm{bps}$ were achieved at a depth of $1000 \mathrm{~m}$. In [79] and [80], a testbed was developed to study the channel behavior for acoustic waves over the string pipes. The results in [79] and [80] have shown that acoustic waves suffer from noticeable dispersion and pipe strings act as a frequency selective channel.

Authors in [70] also performed experiments by using acoustic waves for downhole communications where the data rate of 20 and $6 \mathrm{kbps}$ were achieved for 4.5 and $55 \mathrm{~m}$ depth, respectively. Acoustic waves were generated by using a magnetostrictive actuator which converts electrical signals into acoustic vibrations. The acoustic signals were then transmitted over the drill string to the bottom and received back at the surface by the geophones. For a frequency selective channel of the drill string, orthogonal frequency division multiplexing (OFDM) was used. The drill string was modeled as a series of alternating short and long resonators where each resonator is described by a scattering matrix $\mathbf{S}$. The string is considered as a two port device where $\mathbf{S}_{11}$ and $\mathbf{S}_{22}$ represents the reflections while $\mathbf{S}_{12}$ and $\mathbf{S}_{21}$ measure the transmission of the acoustic signal, respectively. The scattering parameters are obtained as [70]

$$
\mathbf{S}_{11}=\mathbf{S}_{22}=\dot{r}\left(1-\frac{\left(1-\dot{r}^{2}\right) \exp ^{-2 j \gamma \ell}}{1-\dot{r}^{2} \exp ^{-2 j \gamma \dot{r}}}\right),
$$

and

$$
\mathbf{S}_{12}=\mathbf{S}_{21}=1-\frac{\left(1-\dot{r}^{2}\right) \exp ^{-2 j \gamma \ell}}{1-\dot{r}^{2} \exp ^{-2 j \gamma \ell}}
$$

respectively. The terms $\ell$ and $\dot{r}$ represents the length of the segment and the reflection coefficient, respectively, while $\gamma$ is the function of the attenuation coefficient $\alpha$, frequency $f$, and velocity of the acoustic signal $v$ as, $\gamma=\frac{2 \pi f}{v}-j \alpha$. Based on the sub-matrices of $\mathbf{S}$, the $\mathbf{T}$ matrix is written as

$$
\mathbf{T}=\frac{1}{\mathbf{S}_{12}}\left[\begin{array}{cc}
\mathbf{S}_{12} \mathbf{S}_{21} & \mathbf{S}_{11} \\
-\mathbf{S}_{22} & 1
\end{array}\right] \text {. }
$$

The channel frequency response for the whole string is then calculated as

$$
\mathbf{T}_{s}=\prod_{i=1}^{N} \mathbf{T}_{i}
$$

where $i=1,2, \ldots N$ is the number of segments of the drill string. Besides the modeling of drill pipe, arrangements of pipes also play an important role in acoustic communication during drilling. Hence, the authors in [81] argued that ascendto-descend arrangement of pipes provide best telemetry performance for downhole acoustic communication. The problems of acoustic noise and attenuation of the acoustic signal due to the pipes joints were studied in [71]. The authors proposed a single carrier with frequency domain equalization (SCFDE) to improve the reliability of the acoustic transmission along the pipe strings. The impact of multiphase flow was examined in [72] for downhole acoustic communication with amplitude shift keying and frequency shift keying modulation schemes. Moreover, the authors in [73] introduced the use of trellis coded modulation for downhole acoustic communication where a more realistic model of $1000 \mathrm{~m}$ depth was considered with an achievable data rate of up to 400 bps.

Recently, non-contiguous OFDM with adaptive pilot design was used in [78] to provide data rate of up to 500 bps at the depth of $53.76 \mathrm{~m}$. The drill string was modeled to the schematic diagram shown in Fig. 5 where $x$ is the transmitted signal, $y$ is the received signal, $H$ is the channel transfer function, $n_{b}$ is drill bit noise, $n_{e}$ is the environment noise, and $n_{s}$ is the surface noise. Then the received signal is written as

$$
y=H x+H\left(n_{b}+n_{e}\right)+n_{s}=H\left(x+n_{d}\right)+n_{s},
$$


TABLE III: Comparison of acoustic waves-based IoUT.

\begin{tabular}{|c|c|c|c|c|c|}
\hline Ref. & Data rate & Depth & Issue addressed & Applications & Year \\
\hline [11] & - & - & Soil sampling & Agriculture & 2002 \\
\hline$[64],[65]$ & - & - & Soil moisture detection & Agriculture & 2003-2004 \\
\hline [15] & $20 \mathrm{bps}$ & $1120 \mathrm{~m}$ & Down-hole communication & Underground drilling & 2006 \\
\hline [67] & $20-60 \mathrm{bps}$ & $1000 \mathrm{~m}$ & $\begin{array}{l}\text { Field tests for down-hole com- } \\
\text { munication }\end{array}$ & Underground drilling & 2007 \\
\hline [12] & - & - & Soil moisture detection & Agriculture & 2010 \\
\hline [68] & - & - & $\begin{array}{l}\text { Detection of mines using } \\
\text { acoustic waves }\end{array}$ & $\begin{array}{l}\text { Underground mines } \\
\text { detection }\end{array}$ & 2010 \\
\hline [69] & - & - & $\begin{array}{l}\text { Detection of rock deformation } \\
\text { by using acoustic emission }\end{array}$ & Seismic & 2011 \\
\hline [70] & $\begin{array}{l}6 \text { and } 20 \\
\mathrm{kbps}\end{array}$ & 55 and $4.5 \mathrm{~m}$ & $\begin{array}{l}\text { OFDM for down-hole commu- } \\
\text { nication }\end{array}$ & Underground drilling & 2013 \\
\hline [71] & - & - & $\begin{array}{l}\text { Impact of pipe joints on signal } \\
\text { transmission }\end{array}$ & Underground drilling & 2013 \\
\hline [72] & - & - & $\begin{array}{l}\text { Impact of multi-phase flow } \\
\text { with ASK and FSK }\end{array}$ & Underground drilling & 2014 \\
\hline [73] & 400 bps & $1000 \mathrm{~m}$ & $\begin{array}{l}\text { Trellis coded modulation for } \\
\text { down-hole communication }\end{array}$ & Underground drilling & 2014 \\
\hline [66] & - & - & Universal soil loss equation & Agriculture & 2015 \\
\hline$[74]-[76]$ & - & - & Detecting cracks in pipelines & $\begin{array}{l}\text { Underground } \\
\text { pipelines monitoring }\end{array}$ & 2011-2015 \\
\hline [77] & - & - & $\begin{array}{l}\text { Investigation of single channel } \\
\text { and multi-channel accelerome- } \\
\text { ters }\end{array}$ & Down-hole telemetry & 2017 \\
\hline [78] & 500 bps & $53.76 \mathrm{~m}$ & $\begin{array}{l}\text { NC-OFDM for down-hole } \\
\text { communication }\end{array}$ & Underground drilling & 2018 \\
\hline [13], [14] & - & - & $\begin{array}{l}\text { Wireless data transmission in } \\
\text { soil }\end{array}$ & Agriculture & 2018 \\
\hline
\end{tabular}

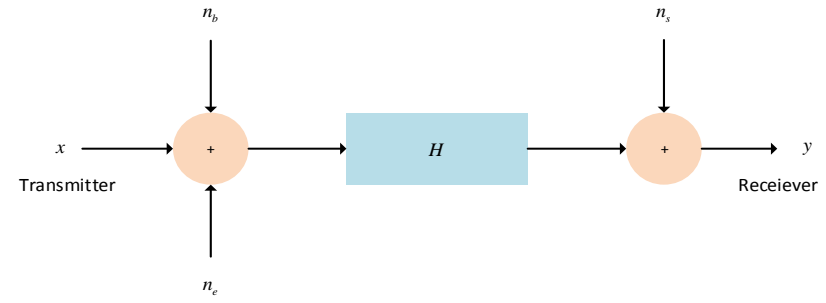

Fig. 5: Equivalent transmission model of the drill string.

where $n_{d}$ is the total transmitter noise. Least square estimation was used in [78] to estimate the acoustic channel characteristics along the drill string.

Moreover, in addition to the channel model for acoustic waves based underground communications, work on the transceivers design for such applications has also been an active area of research. For example, in [82], a novel receiving unit for acoustic communication along the drill string was proposed. Similarly, a tri-axial accelerometer was used in [77] to compare the single channel and multi-channel uphole acoustic communication in oil wells. Nevertheless, Gao et. al studied the transmission of acoustic waves along the drill strings for various applications [83]. Besides, the literature on transmission characteristics and transceiver design, studies on the characteristics of acoustic signals also exists for rock failure [69], cracks in pipelines [74]-[76], and landmines detection [68]. Table III summarizes the literature on acousticbased IoUT.

\section{Mud Pulse Telemetry for IoUT}

The most common and mature method for downhole communication is mud pulse telemetry (MPT) which dates back to the mid of the $19^{\text {th }}$ century. The early MPT systems were able to only communicate the azimuth and inclination information for the wells navigation. The main concept of MPT lies in the circulation of the mud for the transmission of the data [7]. During the drilling process, the pumps at the surface circulate the mud down to the drill string through the pressure pulses [84], [85]. The mud is used to cool the downhole drill string components, carry information from the bottom to the surface, and balance the pressure. The mud passes through a valve which restricts and generates the pressure waves. The pressure pulses are controlled and are used to modulate, frequency, amplitude, and phase of the mud pulse signals [86]. Three different types of mud pulse signals are transmitted; i.e., positive, negative, and continuous wave pulses as shown in Fig. 6 [87]. The signal processing modules at the surface recognize these pressure pulses. The pressure pulse signals in MPT systems are encoded by various techniques to carry the critical information, such as temperature, pressure, and conductivity etc. of the well. Table IV summarizes the literature on MPT systems based IoUT. Although the MPT systems are mature, the mud pulse signals suffers from several impairments which are discussed in the following subsections.

\section{A. Mud Pump Noise}

During the down-link transmission of the mud pulse signal, the piston in the valve moves back and forth to generate the signal. At the same time the up-link signal is generated 


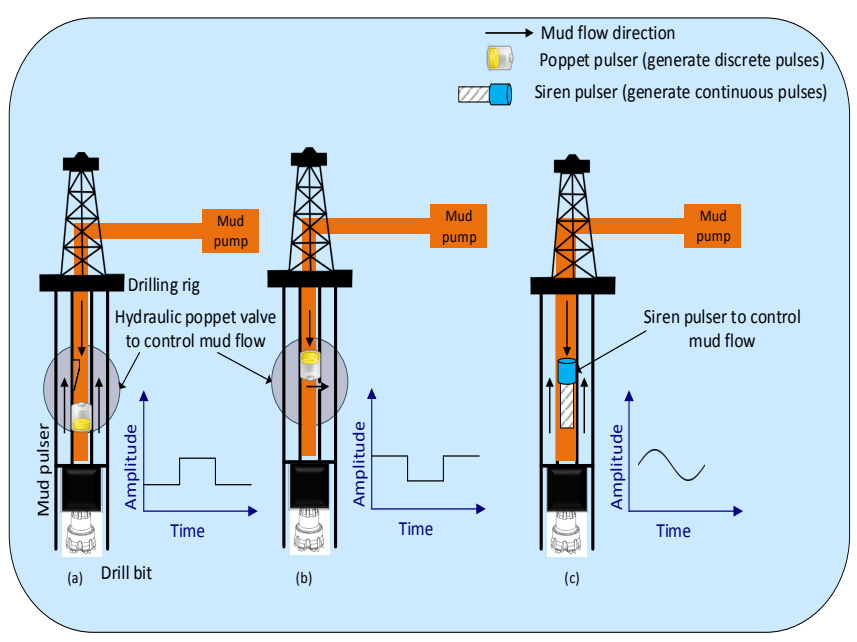

Fig. 6: Three different types of MPT systems. (a) Positive pulses from blocking/unblocking of the fluid; (b) Negative pulses by pressure in the drill string; (c) Continuous pulses by using a rotor.

in the similar fashion in opposite direction resulting in the interference between the down-link and up-link signals [88]. Moreover, the pressure of the pump creates noticeable amount of frequency and amplitude varaitions in the frequency range of 1-20 Hz. To diminish these effects, MPT systems uses two different transducers at the surface receivers which are well spaced [89], [90]. In [91] and [92], the authors have used least mean square filtering algorithm to reduce the noise generated by the mud pumps.

\section{B. Attenuation and Dispersion}

As the mud pulse signals propagate along the borehole, the signals are attenuated and dispersed due to under-balanced drilling mud [93]. The major sources of attenuation are the mud type, joints in the drill string, signal frequency, diameter of the string, and borehole depth [94]. Low frequency signals can be used to avoid excessive attenuation of the mud pulse signals [95].

\section{Rock Fragments and Gas Leakage}

During the drilling process, rock particles and gas may enter the mud used for the pressure pulses which changes the density and compressibility of the mud [96]. These rock particles and gas reduce the transmission speed of the pressure pulse waves. Hence, it is essential to study the velocity continuity of the drilling mud because the gas leakage into the mud can lead to unstable drilling which can cause environmental pollution and potential loss of human lives [97].

\section{Magnetic Induction fOR IoUT}

One of the major factors which limit the evolution of IoUT is the challenging underground environment. We have previously discussed that the heterogeneous soil medium and

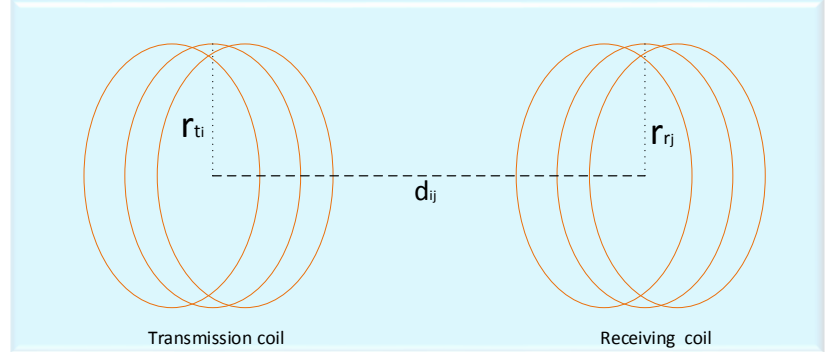

Fig. 7: MI communication link.

water content of the soil limit the transmission range of EMbased IoUT [40]. Hence, magnetic induction (MI) has been introduced to overcome the limitations of EM waves for IoUT [21], [98]. In this section, we will cover multiple aspects of MI-based IoUT which include channel modeling, networking, and localization.

\section{A. Channel Modeling}

MI transceivers consist of induction coils which produce quasi-static magnetic fields that can be sensed by the nearby coil. Moreover, each coil is connected to a capacitor such that the coil operates at a resonance frequency. Unlike the small commercial near field communications (NFC) coils, moderate size of coils are preferable for IoUT to cover long transmission ranges $(10-100 \mathrm{~m})$. A time-varying magnetic field is used in MI-based IoUT for communication between a transmitting and a receiving node. The coil antenna of the transmitting node produces a time-varying magnetic field which induces the current at the coil antenna of the receiver. The design of a conventional MI-based transceiver is shown in Fig. 7 where $d_{i j}$ is the distance between the coil $i$ and $j$, and $r_{t_{i}}$, $r_{r_{j}}$ are their radiuses, respectively. The transmitting current $I=I_{0} e^{-j \omega t}$ with direct current $I_{0}$ and angular frequency $\omega$ induces current in the nearby coil. However, if the transmitting and receiving coils are not well coupled, a single coil may not guarantee communication, and therefore a tri-directional coil structure was proposed in [99] for efficient MI communication (see Fig 8). Based on the MI phenomena, the link budget for MI-communication at high frequency and with a large number of turns in the transmitter coil $N_{t}$ is given in [21], [99]-[102] as

$$
P_{r_{j}}=\frac{\omega \mu P_{t_{i}} N_{r_{j}} r_{t_{i}}^{3} r_{r_{j}}^{3} \sin ^{2} \alpha_{i j}}{16 R_{0} d_{i j}^{6}},
$$

where $\mu$ represents the permeability of the soil, $P_{t_{i}}$ is the transmit power, $N_{r_{j}}$ is the number of turns in the receiver coil, $\alpha_{i j}$ is the angle between the axes of the two coils, and $R_{0}$ is the resistance of a unit length loop. Note that the path loss exponent for MI-based underground is channel is much higher than the free space path loss model. The authors in [99] experimentally validated the link budget expression in (20). Based on the above channel model, different modulation schemes, such as BPSK, QPSK, and QAM were proposed in [103] for MI-based underground communications. Consequently, a square wave with pulse width modulation was used in [104] to provide inductive power transfer and average data 
TABLE IV: Summary of the literature on MPT systems.

\begin{tabular}{|c|c|c|c|c|c|}
\hline Ref. & Frequency & Depth & Issue addressed & Applications & $\overline{\text { Year }}$ \\
\hline [84] & $10-12 \mathrm{~Hz}$ & $1.7 \mathrm{~km}$ & $\begin{array}{l}\text { Generation, transmission, and } \\
\text { reception of mud pulse signals }\end{array}$ & Deep water drilling & 2001 \\
\hline [85] & $30 \mathrm{~Hz}$ & $0.5 \mathrm{~km}$ & $\begin{array}{l}\text { Novel mud pulser which han- } \\
\text { dle the varying nature of the } \\
\text { channel }\end{array}$ & Oil and Gas reservoirs & 2008 \\
\hline [86] & $40 \mathrm{~Hz}$ & - & $\begin{array}{l}\text { Novel method by using a lin- } \\
\text { ear actuator to generate pres- } \\
\text { sure pulses }\end{array}$ & Down-hole telemetry & 2008 \\
\hline [87] & $12-24 \mathrm{~Hz}$ & $150 \mathrm{~m}$ & $\begin{array}{l}\text { Adaptive noise cancellation } \\
\text { technique for the mud pump }\end{array}$ & Underground drilling & 2018 \\
\hline [88] & $1-20 \mathrm{~Hz}$ & - & $\begin{array}{l}\text { Novel decoding technique to } \\
\text { overcome the pump noise, re- } \\
\text { flection noise, and random } \\
\text { noise for MPT systems }\end{array}$ & Underground drilling & 2007 \\
\hline [91] & - & - & Down-hole noise cancellation & Underground drilling & 2008 \\
\hline [93] & - & - & $\begin{array}{l}\text { Novel MPT system for under- } \\
\text { balanced drilling }\end{array}$ & Underground drilling & 2000 \\
\hline [94] & $10-100 \mathrm{~Hz}$ & - & $\begin{array}{l}\text { Investigation of the pressure } \\
\text { wave propagation characteris- } \\
\text { tics }\end{array}$ & $\begin{array}{l}\text { Oil and Gas explo- } \\
\text { ration }\end{array}$ & 2013 \\
\hline [95] & - & - & $\begin{array}{l}\text { Method to detect increase or } \\
\text { decrease in the pressure for the } \\
\text { MPT systems }\end{array}$ & $\begin{array}{l}\text { Oil and Gas explo- } \\
\text { ration }\end{array}$ & 2016 \\
\hline [96] & $\begin{array}{l}10-1000 \\
\mathrm{~Hz}\end{array}$ & - & $\begin{array}{l}\text { Novel hard rock drilling tech- } \\
\text { nique by using abrasive water }\end{array}$ & Underground drilling & 2016 \\
\hline
\end{tabular}

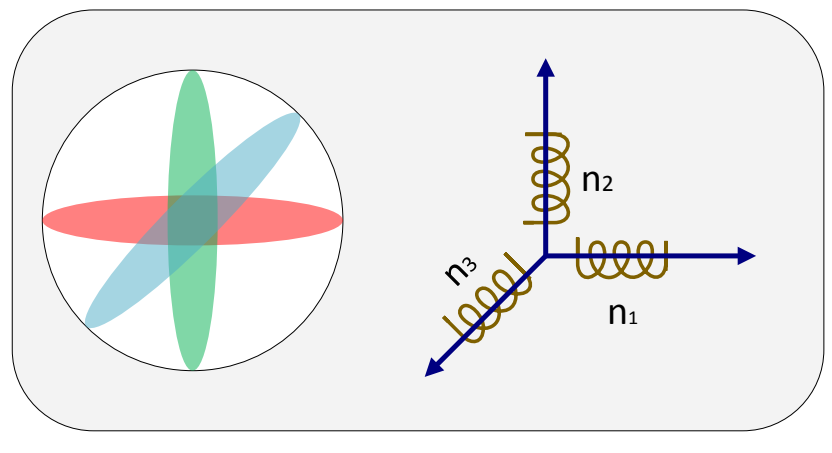

Fig. 8: Tri-directional MI coils.

rate communication for MI-based IoUT. The expression for the magnetic field generated by a coil at distance $d$ was given in [104] as

$$
B=\frac{\mu_{0} I r^{2} n N_{t}}{2\left(d^{2}+r^{2}\right)^{\frac{3}{2}}}
$$

where $\mu_{0}$ is the permeability of free space, $r$ is the radius of the coil, $I$ is the current, and $N_{t}$ is the number of turns of the transmitting coil. At the receiver coil, the electromagnetic field is produced as

$$
E=N_{r} A \omega B \cos \omega t
$$

where $N_{r}$ is the number of turns of the receiving coil, $A$ is the area of the coil, and $t$ is the instantaneous time. Based on the electromagnetic field, the voltage $V$ is measured as follows

$$
V=\frac{E R_{l_{2}}}{R_{l_{2}}+j \omega L+Z_{r}}
$$

where $R_{l_{2}}$ is the resistance of the receiving coil, $L$ is the inductance, and $Z_{r}$ is the load resistance by connecting the parallel capacitance resistance and pre-amplifier circuit (see

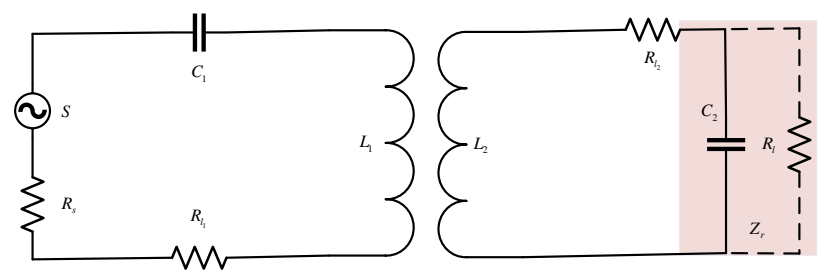

Fig. 9: Equivalent circuit model for MI-based transmitter and receiver.

Fig. 9), i.e,

$$
Z_{r}=\frac{R_{l}}{1+j \omega C_{2} R_{l}} .
$$

Substituting (22) into (23) yields the expression for the measured voltage as follows [105]

$$
V=\frac{N_{r} A \omega B R_{l_{2}}}{R_{l_{2}}+j \omega L+Z_{r}}
$$

Based on the above forumation, the authors in [105] obtained the following received power expression

$$
P_{r}=\frac{V_{0}^{2} k^{2} F_{1}^{2} F_{2}}{2 R_{l_{1}} F_{1_{t}}}
$$

where $V_{0}$ is the voltage source, $k=\frac{M}{\sqrt{L_{1} L_{2}}}, F_{1}=\frac{\omega L_{1}}{R_{l_{1}}+R_{s}}$, $F_{2}=\frac{\omega L_{2}}{R_{l_{2}}}$ are the quality factors of transmitting and receiving coils, respectively, $F_{1_{t}}$ is the instantaneous quality factor, $M=\frac{\mu_{0} \pi N_{t} N_{r} r^{4}}{2\left(\sqrt{d^{2}+r^{2}}\right)^{3}}$ is the mutual induction, and $R_{s}$ is the source resistance. Authors in [105] were also able to determine the size of the antenna and the number of turns in the coil for MI-based low power and low-frequency underground communications. Furthermore, they studied the impact of soil conductivity $\sigma$, on the MI-based underground communication links [106]. Adding $\sigma$ to the channel, modifies the mutual 
inductance $M$ as follows

$$
M_{\sigma}=\frac{\mu_{0} \pi N_{t} N_{r} r^{4}}{2 \pi d^{3}} \exp ^{-\alpha d},
$$

where $\alpha=\frac{1}{\sqrt{\pi f \mu_{0} \sigma}}$ is the attenuation constant. In [107], the soil path attenuation model with the best operating frequency range identification was presented for MI-based IoUT. Also, multiple parallel receiver circuits were used in [107] to achieve higher voltage gain. A pulse power method (use of relay coils) was used in [108] to improve the transmission range of underground MI-based communications. The received power expression in [108] is derived from (26) and is given as

$$
P_{r}=\frac{P_{t} \mu_{0} N_{t} N_{r} r_{t}^{3} r_{r}^{3}}{32 \pi d^{6} C_{1} R_{0}^{2}}
$$

Recently, the authors in [109] suggested meta-material based MI coils for long-range subsurface communications. It was shown in [109] that using of meta-material coil provide better capacity for the same transmission distance. Authors in [110] and [111] evaluated the performance of IoUT for sandy and stone type of media where it was shown that the receiver sensitivity should be $-70 \mathrm{dBm}$. Table V summarizes the literature on the various physical layer issues of MI-based IoUT.

\section{B. Networking}

The typical MI-based IoUT network consists of buried sensors (underground things) and aboveground equipment (surface things) as shown in Fig. 10. Hydraulic fracturing is used to inject the underground things into the well bottom or the reservoir [112], [113]. The surface things can provide extended MI communication link by using large dipole antennas and large transmission power [112]. Therefore, the downlink communication channel is assumed to be single hop while the up-link communication channel is multi-hop due to the limited transmission range of underground things [114]. The surface things can also work as anchors for the localization purpose.

Although MI-based techniques address the issue of dynamic underground channel model, the transmission distance is lower for practical use. In practical applications, the transmission distance of MI-based IoUT is improved by using the relay coils [115]-[118]. In [115], optimal deployment strategies for both one-dimensional and two-dimensional MI-based waveguide was investigated. Additionally, optimal number of relay coils were calculated to minimize the deployment cost. Minimum spanning tree (MST) algorithm was used to minimize the number of relay coils. However, MST algorithm is not robust to the coil displacement and node failure. Therefore, Voronoi-Fermat (VF) algorithm was proposed to improve the robustness without increasing the deployment cost. Besides the use of relay coils, the authors in [116] suggested the use of superconductor and meta-materials, for the MI coil design to improve the transmission range. Authors in [117] used relays to reduce the path loss for MI-based underground communication. Similarly, meta-matrial shell was used for MIbased transceivers to improve their received power [118].

In [119]-[121], the network capacity of a multi-hop MIbased IoUT was evaluated. Throughput of the bottleneck link

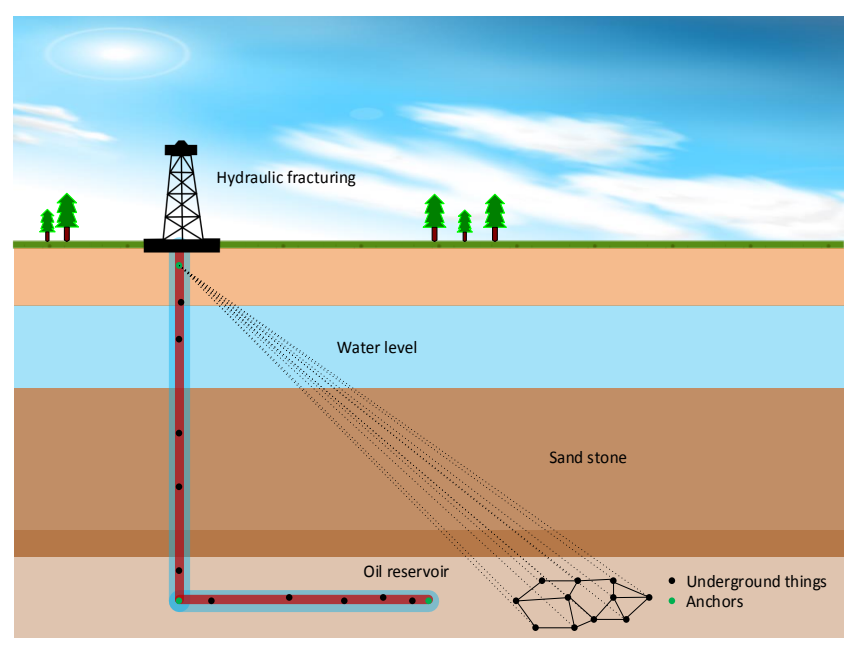

Fig. 10: Network model for MI-based IoUT for oil and gas reservoirs.

which is related to the overall network capacity was investigated in [119]. The polarization of MI coils was exploited to reduce the interference and improve the network capacity. The channel capacity for any arbitrary link $i$ was given in [119] as

$$
C_{i}=\int_{-\infty}^{+\infty} \log _{2}\left(1+\frac{P_{t_{i}}}{L_{i} \cdot \mathrm{E}\left\{P_{n}\right\}}\right) d f
$$

where $P_{t_{i}}$ is the transmit power, $L_{i}$ is the path loss, and $\mathrm{E}\left\{P_{n}\right\}$ is the total noise power spectral density. It was shown that the proper orientation of the coils significantly reduces the interference and improve the network capacity. Similarly, optimal system parameters and topology were proposed in [120] to avoid the bottlenecks in the network and to achieve higher throughput by using MI-based waveguides. Based on the above channel capacity, the throughput of link $i$ was given in [120] as

$$
T_{i}=\frac{C_{i}}{N_{d_{i}}\left(1+N_{\text {int }_{i}}\right)},
$$

where $N_{d_{i}}$ is the number of data streams and $N_{i n t_{i}}$ is the number of interfering nodes. The results in [119] and [120] suggest that the use of relays and optimizing the orientation of the coils improve the throughput of a multi-hop MI-based IoUT network. Nevertheless, digital transmission schemes were introduced in [121] for both direct and multi-hop MIbased underground links. Frequency division multiplexing was suggested to equalize the long channel impulse response. Moreover, various modulation schemes, such as BPSK and QAM were investigated for multi-hop MI links, and it was suggested that higher order modulations could be avoided by using a bandwidth expansion mechanism.

A distributed environment aware cross-layer protocol (DEAP) was proposed in [122] to satisfy the quality of service (QoS) requirement, achieve higher throughput, and reduce energy consumption. For a given MI-based underground link $i-j$, the DEAP protocol maximizes the QoS, $Q_{i j}$, and minimizes the energy consumption, $E_{i j}=U\left(\frac{P_{i j}}{R_{i j} R_{c_{i j}}}+2 E_{b}\right)$, 
TABLE V: Various physical layer issues addressed in the literature for MI-based IoUT.

\begin{tabular}{|c|c|c|c|}
\hline Ref. & Frequency & Issue addressed & Design aspect \\
\hline [21] & 300 and $900 \mathrm{MHz}$ & Underground channel modeling for MI & Channel modeling \\
\hline [99] & 0.02 and $30 \mathrm{MHz}$ & $\begin{array}{l}\text { Use of tri-directional MI coils for omni-directional coverage } \\
\text { and waveguides to improve the transmission range }\end{array}$ & $\begin{array}{l}\text { Channel modeling and test-bed de- } \\
\text { velopment }\end{array}$ \\
\hline [100] & - & $\begin{array}{l}\text { Discuss various issues for underground MI-based communi- } \\
\text { cation }\end{array}$ & Cross-layer solutions \\
\hline [101] & 10 and $300 \mathrm{MHz}$ & $\begin{array}{l}\text { Path loss and bandwidth analysis for underground MI com- } \\
\text { munications }\end{array}$ & Channel modeling \\
\hline [102] & $100 \mathrm{kHz}$ & Path loss and capacity measurement for underground MI link & Channel modeling \\
\hline [103] & & BPSK, QPSK, and QAM for the underground MI links & Modulation schemes \\
\hline [104] & $246 \mathrm{kHz}$ & $\begin{array}{l}\text { Use of pulse width modulation for underground MI-based } \\
\text { communication }\end{array}$ & Testbed development \\
\hline [105] & $246 \mathrm{kHz}$ & Link budget calculation for underground MI link & Path loss modeling \\
\hline [106] & $5 \mathrm{kHz}$ & Impact of soil conductivity on the underground MI link & Channel modeling \\
\hline [107] & $75 \mathrm{kHz}-30 \mathrm{MHz}$ & Soil path attenuation model and best frequency selection & $\begin{array}{l}\text { Channel modeling and testbed de- } \\
\text { velopment }\end{array}$ \\
\hline [108] & $300-900 \mathrm{MHz}$ & $\begin{array}{l}\text { Improving transmission range by using relays and achieving } \\
\text { higher voltage gain with multiple parallel receiver circuits }\end{array}$ & $\begin{array}{l}\text { MI-based multi-hop underground } \\
\text { communication }\end{array}$ \\
\hline [109] & $20-50 \mathrm{MHz}$ & $\begin{array}{l}\text { Meta-material for coil design to improve transmission range } \\
\text { and capacity }\end{array}$ & Coil design \\
\hline [110], [111] & $10 \mathrm{MHz}$ & To study the impact of different medium on the MI link & Testbed development \\
\hline
\end{tabular}

where $\frac{P_{i j}}{R_{i j} R_{c_{i j}}}$ is the distance-dependent energy required to transmit a single bit, and $E_{b}$ is the electrical circuit energy required for a single bit transmission. $R_{i j}$ is the transmission rate, $R_{c_{i j}}$ is the channel coding rate, and $U$ is the length of the packet. This is a minimization-maximization problem where energy needs to be minimized, and the QoS needs to be maximized. Therefore, a cross-layer optimization strategy was used to save the energy and improve the network throughput. A two-stage cross-layer protocol called Xlayer was proposed in [123] for multi-hop MI-based IoUT to guarantee the QoS requirement. Xlayer protocol was able to achieve high throughput, low delay, and low energy consumption. A fullduplex meta-material enabled MI-based communication was proposed in [124] to reduce the transmission delay for multihop underground communication.

Recently, connectivity analysis for IoUT was provided in [125] where the probability of a connected network increases with an increase in the number of underground things and low volumetric water content. Additionally, the connectivity performance of the EM and MI-based IoUT network was compared where the results have shown that for low volumetric water content (VWC) of the soil (1\%), the connectivity of EM and MI-based IoUT is similar. However, for the same node density when the VWC of the soil is increased to $5 \%$, the EM-based network becomes unconnected while the MI still maintains the connectivity. Table VI presents the literature on various network layer issues for MI-based IoUT.

\section{Localization}

Localization is an essential task in wireless networks which enable various location-based services. Hence, localization techniques for the terrestrial and underwater wireless communication networks are well investigated in the past. For example in [137] the authors reviewed various localization techniques for terrestrial wireless networks. Similarly, in [138] localization techniques for marine networks are studied. The localization techniques can be classified based on the ranging technique (range-based/range-free), type of computation (centralized/distributed), and space (2D/3D). However, the literature on localization techniques for the underground wireless networks is limited due to various challenges, such as harsh and light-less underground environment, non-availability of global positioning system (GPS) signals, high attenuation, and narrow operational area. Although efforts have been made in the past to develop localization techniques for harsh environments, such as indoor and underwater, however, the underground environment does not support the use of these communication technologies, and therefore the localization techniques for the indoor and marine environment cannot be directly applied to the underground case [23].

Accordingly, a two-dimensional (2D) localization technique was developed by Andrew et. al in [126] by using magneticinduction to track animals underground. Furthermore, they extended their 2D tracking system to a three-dimensional (3D) one in [127]. Moreover, they used the 3D MI-based tracking model for underground rescue operations [128]. As the propagation of MI signals is highly affected by the soil medium, therefore, the impact of minerals and rocks on the localization accuracy was studied in [129]. It was observed in [129] that the skin effect of the underground medium is almost negligible at very low frequencies whereas the localization accuracy depends on the attenuation properties of various underground materials. Recently, simulated annealing wasn used for MI-based terrestrial and underground wireless networks in [130], [131] which can achieve sub-meter level accuracy.

Furthermore, a modified semidefinite programming-based relaxation technique was used in [132] to determine the position of the underground sensors. A single anchor was used in [133] to find the location of all other underground sensors in 3D for MI-based IoUT. Trilateration, machine learning, and hybrid passive localization techniques were used in [134] to estimate the position of a target node in 2D MIbased IoUT. Recently, an analytical model has been presented in [135] for distance estimation in MI-based IoUT. The achievable accuracy of localization techniques is characterized 
TABLE VI: Various network layer issues addressed in the literature for MI-based IoUT.

\begin{tabular}{|c|c|c|c|}
\hline Ref. & Frequency & Issue addressed & Design aspect \\
\hline$[115]$ & $10 \mathrm{MHz}$ & $\begin{array}{l}\text { Improvement of the transmission range and robustness, and } \\
\text { selecting optimal number of relays }\end{array}$ & Deployment strategies \\
\hline [116] & - & $\begin{array}{l}\text { Improvement of the transmission range by using relays and } \\
\text { meta-materials }\end{array}$ & $\begin{array}{l}\text { Multi-hop networking/Hardware } \\
\text { design }\end{array}$ \\
\hline [117] & $300-900 \mathrm{MHz}$ & Improvement of the transmission range by using relays & Deployment strategies \\
\hline [118] & $10 \mathrm{kHz}$ & $\begin{array}{l}\text { Use of meta-material shell for the transceiver design to } \\
\text { improve the received power }\end{array}$ & Transceiver design \\
\hline [119] & - & $\begin{array}{l}\text { Investigating the effect of coil orientation and polarization on } \\
\text { the channel capacity }\end{array}$ & Interference minimization \\
\hline [120] & 2 and $2.5 \mathrm{MHz}$ & Throughput optimization & $\begin{array}{l}\text { Multi-hop networking and interfer- } \\
\text { ence minimization }\end{array}$ \\
\hline [121] & - & $\begin{array}{l}\text { Optimization of system parameters for multi-hop underground } \\
\text { MI links }\end{array}$ & Maximizing the data rate \\
\hline [122] & $7 \mathrm{MHz}$ & $\begin{array}{l}\text { Improving throughput, reducing energy consumption and time } \\
\text { delay }\end{array}$ & Cross layer protocol \\
\hline [123] & - & Throughput, delay, and energy consumption analysis & Cross layer protocol \\
\hline [124] & $10 \mathrm{MHz}$ & $\begin{array}{l}\text { Transmission range enhancement by using meta-material } \\
\text { based relay coils }\end{array}$ & Transceiver design \\
\hline [125] & $300-1300 \mathrm{MHz}$ & Connectivity analysis of multi-hop MI-based IoUT & Transceiver design \\
\hline
\end{tabular}

TABLE VII: Summary of the literature on localization for MI-based IoUT.

\begin{tabular}{|c|c|c|c|c|}
\hline Ref. & Frequency & Issue addressed & Dimension & Application \\
\hline$[126]$ & $130 \mathrm{kHz}$ & Development of MI-based 2D underground tracking system & $2 \mathrm{D}$ & $\begin{array}{l}\text { Tracking of } \\
\text { underground animals }\end{array}$ \\
\hline [127] & $125 \mathrm{kHz}$ & Testbed for MI-based 3D underground tracking & 3D & Underground mining \\
\hline [128] & $125 \mathrm{kHz}$ & Testbed for MI-based 3D underground tracking & 3D & $\begin{array}{l}\text { Underground rescue } \\
\text { operations }\end{array}$ \\
\hline [129] & $\begin{array}{l}1 \mathrm{kHz}, 100 \mathrm{kHz} \text {, and } \\
10 \mathrm{MHz}\end{array}$ & $\begin{array}{l}\text { Investigating the impact of minerals and rocks on the local- } \\
\text { ization accuracy }\end{array}$ & 3D & $\begin{array}{l}\text { Underground monitor- } \\
\text { ing }\end{array}$ \\
\hline [130], [131] & & $\begin{array}{l}\text { Closed form solution for the distance estimation based on MI } \\
\text { channel }\end{array}$ & 3D & $\begin{array}{l}\text { Underground monitor- } \\
\text { ing }\end{array}$ \\
\hline [132] & $7 \mathrm{MHz}$ & $\begin{array}{l}\text { Using of semi-definite programming for MI-based under- } \\
\text { ground localization }\end{array}$ & $3 \mathrm{D}$ & $\begin{array}{l}\text { Oil and Gas reservoirs } \\
\text { monitoring }\end{array}$ \\
\hline [133] & $10 \mathrm{MHz}$ & $\begin{array}{l}\text { MI-based underground localization by using a single anchor } \\
\text { node }\end{array}$ & $3 \mathrm{D}$ & $\begin{array}{l}\text { Underground monitor- } \\
\text { ing }\end{array}$ \\
\hline [134] & $1 \mathrm{MHz}$ & $\begin{array}{l}\text { Machine learning approach for MI-based underground target } \\
\text { localization }\end{array}$ & $2 \mathrm{D}$ & $\begin{array}{l}\text { Underground rescue } \\
\text { operations }\end{array}$ \\
\hline [135] & - & $\begin{array}{l}\text { Analytic model for distance estimation in MI-based under- } \\
\text { ground communications }\end{array}$ & 2D & $\begin{array}{l}\text { Underground monitor- } \\
\text { ing }\end{array}$ \\
\hline [136] & 7 and $13 \mathrm{MHz}$ & $\begin{array}{l}\text { Analytical expression for the achievable accuracy of MI-based } \\
\text { underground communications }\end{array}$ & 3D & $\begin{array}{l}\text { Oil and Gas reservoirs } \\
\text { monitoring }\end{array}$ \\
\hline
\end{tabular}

by estimation bounds, such as the Cramer Rao lower bound (CRLB). In the past, CRLB have been derived for various wireless networks, such as internet of things (IoT) [139], vehicular ad-hoc networks [140], source localization [141], radar tracking [142], cognitive radio networks [143]-[145], and underwater wireless networks [146]-[148]. Therefore, in [136], we derived the expression of the CRLB for the MIbased IoUT localization. The derived bound in [136] takes into account the channel and network parameters of MI-based IoUT. We consider a 3D oil and gas reservoir setup where the MI coils are injected to the reservoir by using hydraulic fracturing. We assume that the coils are uniformly distributed in a $15 \times 15 \mathrm{~m}^{2}$ fracturing area and the depth of fracture is $1.8 \mathrm{~km}$. Additionally, the buried smart objects are able to communicate with the anchors on the surface in a multi-hop fashion [132]. Table VIII presents the simulation parameters which are mainly taken from [132].

Fig. 11 shows the impact of the number of turns in the MI coil and the noise variance on the achievable accuracy. The values of the noise variance are kept as $0.05,0.3$, and $0.7 \mathrm{~m}$, respectively whereas the frequency is $13 \mathrm{MHz}$. Fig. 11
TABLE VIII: Simulation parameters

\begin{tabular}{|l|l|}
\hline \hline Parameters & Values \\
\hline Frequency & $13 \mathrm{MHz}$ \\
Area of the Fracture & $15 \times 15 \mathrm{~m}^{2}$ \\
Depth & $1.8 \mathrm{~km}$ \\
Radius of the coils & $0.01-0.04 \mathrm{~m}$ \\
Number of turns in coils & $10-30$ \\
Transmit power & $100-200 \mathrm{~mW}$ \\
Unit length resistance of antenna & $0.01 \Omega / m$ \\
Temperature & $418 \mathrm{~K}$ \\
Noise variance & $0.1-0.8 \mathrm{~m}$ \\
Number of underground things & 60 \\
\hline \hline
\end{tabular}

suggests that to get better localization accuracy more number of turns in the coil are required however increasing the number of turns may increase the size of the coil. However, the harsh underground environment requires a small size of the MI coil. Hence, the impact of coil size is examined in Fig. 12 which suggests that increasing the size of the coil improve the accuracy. Thus, there is a trade-off between the size of the coil and the localization accuracy which should be taken into account before the deployment. 


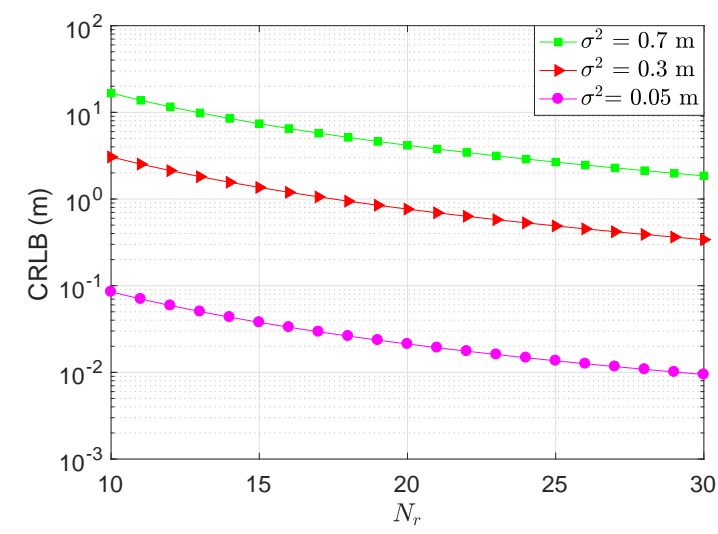

Fig. 11: CRLB vs. Number of turns in the receiver.

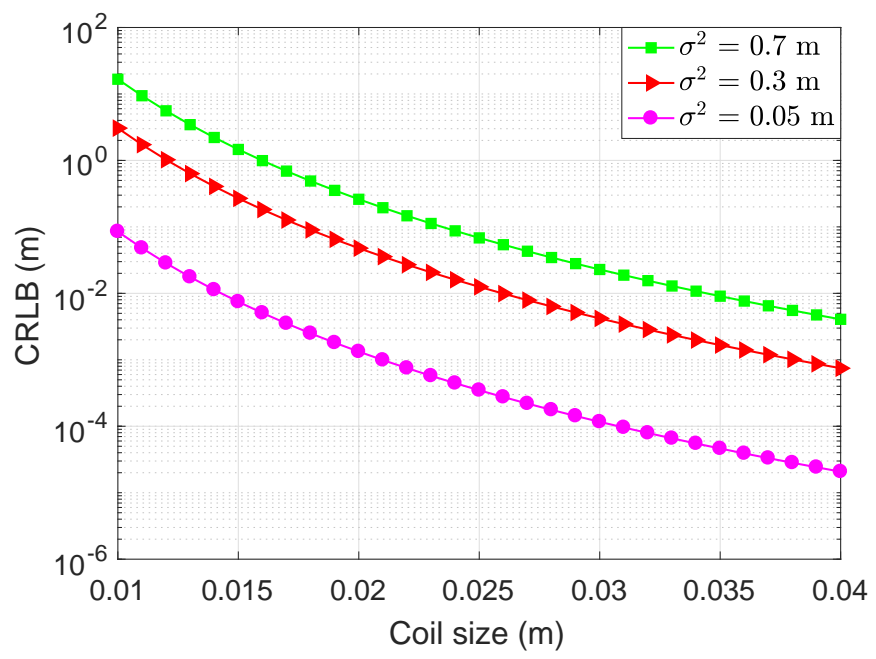

Fig. 12: CRLB vs. Coil size.

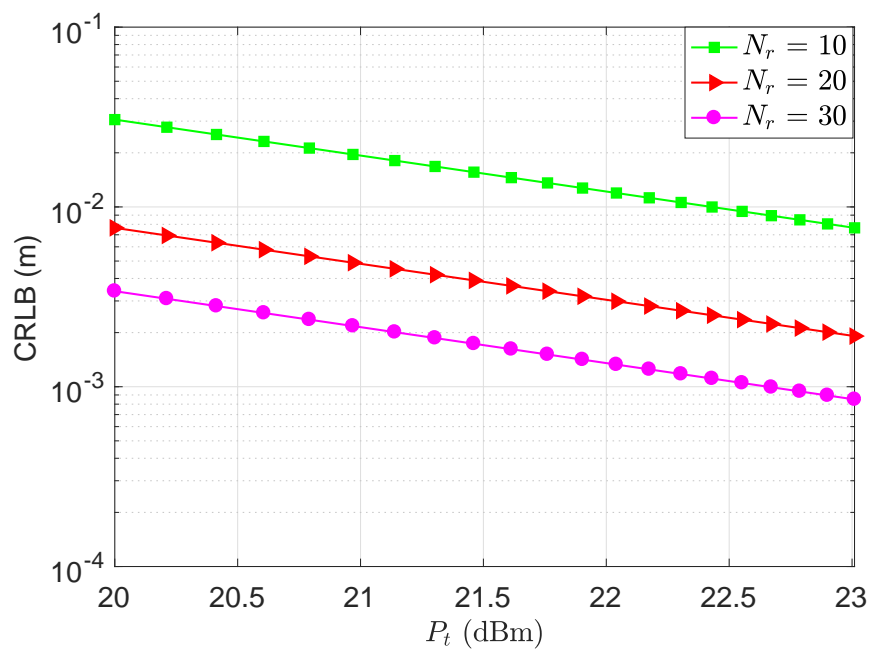

Fig. 13: CRLB vs. Transmit power.

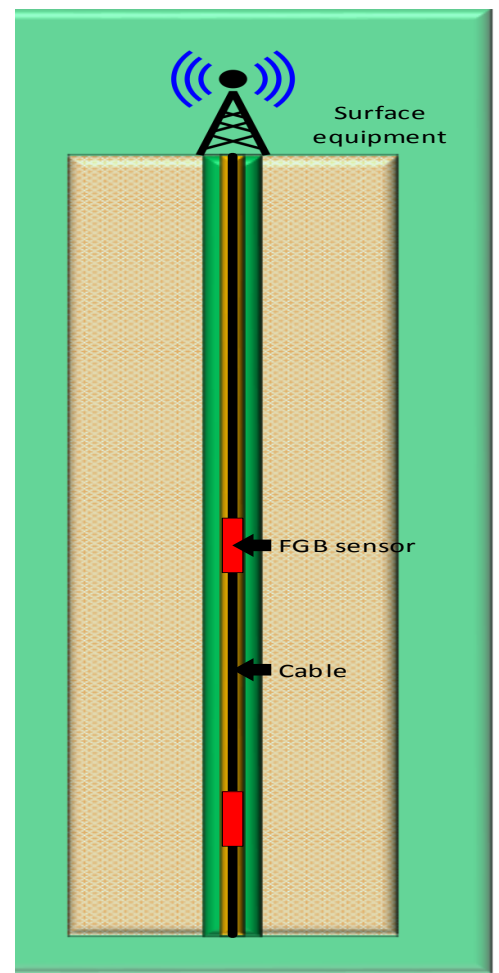

Fig. 14: Fiber optic monitoring system for IoUT.

Moreover, we have also tested the impact of transmission power on the achievable localization accuracy in Fig. 13. Commercially available MI coils have transmission power in the range of $100-200 \mathrm{~mW}(20-23 \mathrm{dBm})$. Thus we kept the transmit power in the range of $20-23 \mathrm{dBm}$ with the variable size of the coils. Fig. 13 shows that with the increase in transmit power the achievable accuracy improves. Accordingly, the above results suggest that the achievable accuracy of any localization algorithm for MI-based IoUT is the function of the number of turns in the MI coil, noise variance, size of the coil, transmit power, frequency, and the number of anchors. Therefore, all these parameters are important to design a robust and accurate localization technique for IoUT.

\section{Charging of the MI Coils}

Lifetime is an important parameter for IoUT due to the harsh underground environment. Therefore, research efforts have been made to improve the lifetime of the IoUT [149]. A charging method for IoUT has been proposed in [149] where a virtual magnetic relay network and optimized routing protocol was used to reduce the energy consumption. However, the charging efficiency of the proposed system in [149] remains very low for even moderate size of coils. Consequently, in [150] an optimized energy model framework was proposed for linear topology. The problem of charging underground coils is still open research problem and other options, such as energy harvesting can be investigated.

\section{WIRED COMMUNICATIONS FOR IOUT}

Wireless communication channels reduce the complexity and cost of underground monitoring. However, existing wire- 
less technologies fail to provide timely, reliable and accurate solutions, especially for the deep underground monitoring. Hence, wired technologies, such as coaxial cable and optical fiber are used for down-hole monitoring [8]-[10], [151], [152]. In 2003, Intelliserv provided a high speed down-hole telemetry system based on coaxial cable as an alternative to the low speed MPT and electromagnetic systems [153]. This system provided real time bi-directional communication at the data rate of 57,000 bits per second which has greatly enhanced the bandwidth for down-hole communication. Coaxial-based system consisted of inductive coils and repeaters along the high strength coaxial cable [9]. Similarly, a fiber optic-based temperate sensing system was developed in [10] to monitor the oil and gas reservoirs. The authors in [151] developed a down-hole communication system in the presence of high temperature and pressure. The proposed system in [151] was based on the SNR characterization of the whole channel where optimal frequency for the transmission is selected. Since for higher data rates, optical fiber has replaced co-axial based wired communication. In this section, we cover the literature on optical fiber-based communication systems for underground applications.

Fiber optic sensing technologies have been applied to several commercial and industrial application in the past two decades. These sensing technologies can provide both sensing and information transfer in the harsh environment. Due to these attributes, they are well suited for the harsh environment in oil and gas reservoirs [154]. In [154], the authors discussed the deployment of underground fiber optic-based monitoring systems, including the assembly of down-hole sensors, data measurement, and installation of the sensors. The authors in [154] also briefly discussed about the installation of the pressure and temperature sensing system in a land well and in the Gulf of Mexico. It was concluded in [154] that fiber Bragg grating (FBG) sensors offer additional advantages, such as high flexibility, high stability, multi-point sensing, and their intrinsic nature to the fiber. The fiber optic based underground sensing system consists of FBG sensors which are connected to the optical fiber cable with the help of ultraviolet photoinscription method [155] (see Fig. 14).

The authors in [159] proposed a FBG based real-time temperature and fluid monitoring system of oil bore-holes. The FBG-based sensor converts the fluid pressure by means of a mechanical transducer into fiber optic strain. The strain of the transducer was given as [159]

$$
s=\frac{s_{l}-\lambda\left(s_{r}+s_{t}\right)}{E},
$$

where $s_{l}, s_{r}, s_{t}$ are the longitudinal, radial, and tangential stresses, respectively, $\lambda$ is the Poisson number, and $E$ is the Young's modulus. Based on the inner and outer radii of the tube, $a_{i}$ and $a_{o}$, the above expression is re-written as

$$
s=\frac{p a_{i}^{2}(1-2 \lambda)}{E\left(a_{o}^{2}-a_{i}^{2}\right)},
$$

where $p$ is the pressure. In [156], Yan et. al designed FBG based seismic geophone for oilfield exploration which showed better sensitivity than the conventional geophones for 10-70
$\mathrm{Hz}$ range of frequencies. Moreover, field tests were performed in [156] where the FBG-based seismic geophone was more immune to the EM interference, and had less non-linear distortion as compared to the conventional FBG sensors.

Wavelength division multiplexing was used in [157] to combine the information from two fibers onto a single fiber for the temperature and pressure sensing in a well-bore. The simultaneous sensing of pressure and temperature, is a cost-effective solution. Experimental results were provided in [157] showing that the proposed multiplexed approach is stable and accurate. Recently, the authors in [158] installed an optical fiber-based down-hole monitoring system at the shoreline of Marmara sea in Turkey to provide geophysical observations. In short, optical fiber based underground monitoring systems provide high-speed communication and are immune to electromagnetic interference which mainly depends on the development of fiber grating sensors [160]. Table IX summarizes the major contributions on wired communications for IoUT.

\section{Visible LIGHT COMMUNICATIONS FOR IOUT}

Light cannot pass through soil therefore visible light communication (VLC) can only be used for down-hole monitoring in gas fields. To the best of the author's knowledge, the only VLC-based down-hole monitoring systems were proposed in [161] and [22]. In [161], light emitting diodes (LEDs) were used at the bottom of the pipeline, and a single photon avalanche diode (SPAD) was used as a receiver at the surface (see Fig. 15). The proposed system in [161] was able to achieve the data rate of 1-5 kbps for the depth of $4000 \mathrm{~m}$. An array of SPAD receivers were set at the surface of the pipe where the number of photons received were estimated as follows:

$$
N_{p}=C_{e} \sum_{n=0}^{R} \frac{(\beta+1) A P_{t}}{2 \pi d^{2} E_{p}} \cos ^{\beta}(\phi(n)) C_{p},
$$

where $C_{e}$ is the photon detection efficiency, $A$ is the area of the SPAD, $d$ is the distance, $\phi$ is the angle of radiation, $P_{t}$ is the average power of LED, $E_{p}$ is the energy per photon, $R$ is the total number of reflections, $C_{p}$ is the reflectivity of pipe, and $\beta=\frac{-\ln (2)}{\ln (\cos (\theta))}$, where $\theta$ is the half power beamwidth. Modulation scheme of non-return-to-zero on-off keying (NRZOOK) was used where binary " 1 " is represented by a high positive voltage and binary " 0 " is represented by a low voltage. Poisson distribution was used in [161] to model the noise where the cumulative distribution function $(\mathrm{CDF})$ is given as follows:

$$
P_{c}(x, \lambda)=\exp ^{-\lambda} \sum_{i=0}^{x} \frac{\lambda^{i}}{i !}
$$

where $\lambda$ is the mean value of the distribution. The probability of error is calculated from the above CDF as follows:

$$
P_{E}=\frac{1}{2}\left[1-P_{c}\left(N_{t}, N_{0}\right)+P_{c}\left(N_{t}, N_{1}\right)\right],
$$

where $N_{t}=\frac{N_{0}+N_{1}}{2}$ is the threshold value, $N_{0}$ is the average number of photons when binary " 0 " is transmitted, and $N_{1}$ is the average number of photons when binary " 1 " is transmitted. It was concluded in [161] that the BER improves 
TABLE IX: Summary of the literature on wired communications for the IoUT.

\begin{tabular}{|c|c|c|c|c|}
\hline Ref. & Type & Issue addressed & Application & Year \\
\hline$[8]$ & Optical fiber & Study on the use of optical fiber for Oilfield industry & Oilfield monitoring & 2002 \\
\hline [9] & Coaxial cable & Development of high speed down-hole communication system & Down-hole telemetry & 2008 \\
\hline [10] & Optical fiber & Down-hole communication temperature sensing & $\begin{array}{l}\text { Management of oil and } \\
\text { gas reservoirs }\end{array}$ & 2010 \\
\hline [151] & Coaxial cable & $\begin{array}{l}\text { Down-hole communication in the presence of high pressure } \\
\text { and high temperature }\end{array}$ & $\begin{array}{l}\text { Management of oil reser- } \\
\text { voirs }\end{array}$ & 2013 \\
\hline [152] & Optical fiber & Discussion on various applications of fiber optic sensing & Underground monitoring & 2018 \\
\hline [154] & Optical fiber & Development of fiber optic based down-hole telemetry system & Down-hole monitoring & 2001 \\
\hline [155] & Optical fiber & $\begin{array}{l}\text { Review of fiber Bragg grating sensors for down-hole moni- } \\
\text { toring }\end{array}$ & Down-hole monitoring & 2000 \\
\hline [156] & Optical fiber & Field tests by using FBG-based seismic geophones & $\begin{array}{l}\text { Oil and Gas reservoirs } \\
\text { monitoring }\end{array}$ & 2007 \\
\hline [157] & Optical fiber & Multiplexing of temperature and pressure FBG sensors & $\begin{array}{l}\text { Oil and Gas reservoirs } \\
\text { monitoring }\end{array}$ & 2012 \\
\hline [158] & Optical fiber & FBG sensors-based testbed development & Geophysical observations & 2015 \\
\hline
\end{tabular}

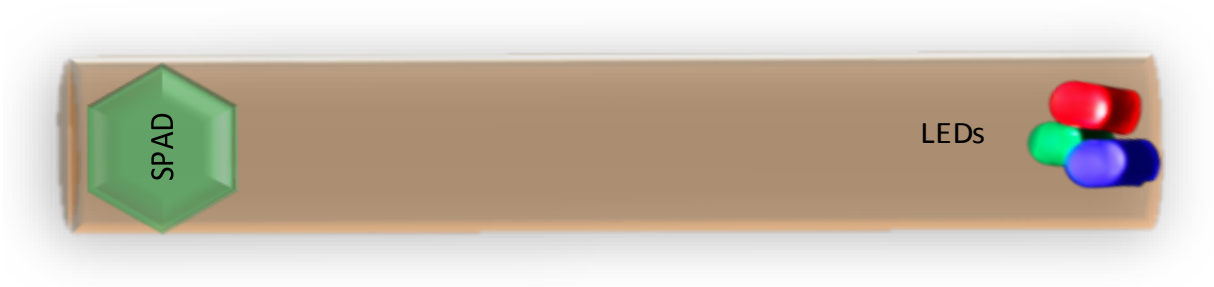

Fig. 15: VLC based IoUT for gas pipelines.

with reducing the half power beamwidth angle and increasing the LED's power. Nevertheless, the authors in [161] assume empty pipeline which is not realistic. Hence, in [22] the authors provided a channel model for VLC-based underground gas pipelines in the presence of methane gas. Ray tracing technique was used to model the channel. The channel impulse response (CIR) for the ray tracing technique is expressed as [22]

$$
h(t)=\sum_{i=1}^{N_{r}} P_{i} \delta\left(t-\tau_{i}\right)
$$

where $N_{r}$ is the number of rays, $P_{i}$ is the power from $i$-th ray, $\tau_{i}$ is the propagation time of $i$-th ray, and $\delta(\cdot)$ is Dirac delta function. By taking the Fourier transform of the CIR yields the frequency response of the optical channel as follows:

$$
H(f)=\int \sum_{i=1}^{N_{r}} P_{i} \delta\left(t-\tau_{i}\right) \exp ^{-j 2 \pi f t} d t
$$

Based on $H(f)$, the frequency response is commonly modeled as

$$
H_{L}(f)=\frac{1}{1+\frac{j f}{f_{c}}},
$$

where $f_{c}$ is the cutt-off frequency. By taking into account the characteristics of LED, the effective channel response in frequency domain is expressed as $H_{e}(f)=H_{L}(f) H(f)$. Based on the expression of the channel response, the BER is given as

$$
\mathrm{BER}=\frac{2(M-1)}{M \log _{2}(M)} Q\left(\frac{1}{M-1} \sqrt{\frac{\left(P_{t} h_{e} r_{s}\right)^{2} T_{s}}{N_{o}}}\right),
$$

where $M$ is the constellation size for $M$-ary PAM schemes, $h_{e}(t)=F^{-1}\left(H_{e}(f)\right), P_{t}$ is the average optical power transmitted, $r_{s}$ is the responsivity of photodetector, $T_{s}$ is the sampling interval, $N_{o}$ is noise power spectral density, and $F^{-1}(\cdot)$ is the inverse Fourier transform. Different pulse amplitude modulation (PAM) schemes were tested, and the results have shown that for a target BER of $10^{-6}, 8$-PAM can reach the target distance of $22 \mathrm{~m}$. Similarly, higher order PAM provides a better data rate but reduced transmission range with a minimum of $3.82 \mathrm{~m}$ for 512-PAM. Nevertheless, the research on VLC-based IoUT is in its infancy and need to be examined in the future.

\section{Future Research Challenges}

The recent advances in IoUT have broadened the scope of this research area. Hence, in this section, we provide various new challenges for the IoUT. Table $\mathrm{X}$ presents the significance of each research challenge for a specific underground application.

\section{A. Deployment}

The deployment of smart objects for IoUT in the harsh underground environment is a challenging issue [23]. Installation and management of smart objects underground are much more difficult compared to the terrestrial networks. Moreover, the underground objects can be easily damaged during the digging process. Hence, efficient deployment of the smart objects is required to minimize the installation cost of the IoUT. For example, a smart object with high energy requirement should be deployed near the surface for ease of management as the replacement of batteries in the underground environment is 
TABLE X: Significance of each research challenge for various applications.

\begin{tabular}{|l|l|l|l|}
\hline \hline Research Challenge & Agriculture & $\begin{array}{l}\text { Seismic } \\
\text { exploration }\end{array}$ & $\begin{array}{l}\text { Oil \& } \\
\text { Gas }\end{array}$ \\
\hline Deployment & Medium & High & High \\
\hline Channel modeling & Medium & Medium & High \\
\hline Transmission range & Low & High & Medium \\
\hline Latency & Low & Low & Medium \\
\hline Reliability & Low & Medium & High \\
\hline Security & Medium & High & High \\
\hline Scalability & Low & Medium & Medium \\
\hline Robustness & Low & Medium & High \\
\hline Networking & High & Medium & Medium \\
\hline Cloud computing & High & Medium & Low \\
\hline Fog computing & Low & Medium & High \\
\hline Localization & Medium & High & Medium \\
\hline \hline
\end{tabular}

challenging. Moreover, to avoid the replacement of batteries, a battery with high capacity should be used, and power saving protocols should be applied. Deployment of underground objects is more challenging in case of seismic exploration and underground drilling as compared to the agriculture applications due to high depth. Hence, various efforts are made in the past based on different metrics and parameters for the optimal deployment of underground objects. For example, in MI-based IoUT, the orientations and polarization of the coils are managed to reduce the power reflections [115]. Similarly, horizontal and vertical deployment strategies were introduced in [119] to reduce the complexity of the network. Moreover, Voronoi tessellation was used in [120] to select the underground objects to be connected to the relay network. Besides, the effect of heterogeneous soil on the path loss also needs to be taken into account for the optimal deployment of underground objects. Unfortunately, the research work on the efficient deployment of IoUT is limited, which takes into account the various system and network parameters, such as deployment depth, number of smart objects, lifetime, and routing.

\section{B. Channel Modeling}

In terrestrial communications, the strength of the EM signal decays with the square of the distance while in the soil, the decay is much faster due to attenuation from the soil medium [40]. The major loss factors for a given frequency in the soil are the permittivity and conductivity of the type of soil. Hence, MI-based communication was introduced for the IoUT which is based on magnetic field propagation [99]. MI-based underground communications is also affected highly by the heterogeneous soil medium. However, most of the existing works assume homogeneous soil medium which is not realistic. In heterogeneous soil medium, the magnetic fields are attenuated differently at each layer of the soil. Therefore, the authors in [33] defined different scaling factors for various depths. Similarly, the signal propagation through the heterogenous medium was analyzed in [162] where the equivalent skin depth was calculated for each layer of soil. The path loss for a single link in MI-based underground communications is characterized in [101]. Moreover, asymmetric transceivers was introduced in [109] to provide long range underground communications in case of misalignment between the MI coils. Although the path loss for each type of communication channel has been extensively analyzed in the past, unfortunately, few efforts have been made to provide a fully functional wireless solution for IoUT with practical signal transmission schemes to verify the channel models. Nevertheless, the demanding applications of the underground stimulate the research in this direction.

\section{Limited Transmission Range}

The MI technology has certain advantages, such as prone to multi-path fading and boundary effects which makes it ideal for the communication and localization in the underground environment [99]. However, MI technology also has some disadvantages; the most significant one is the limited transmission range due to high path loss in the soil. Few efforts have been made in the past to improve the transmission range for MI-based underground communications. For example, the use of relay coils was proposed in [115] and [116] to extend the transmission range. Also, the use of superconductor and metamaterials was recommended in [116] and [118] to improve the transmission range of MI-coils. Besides that, large coils with high transmission power were used for the long-range downlink communication and localization in [132], however, it may not be a practical solution. Hence, the limited transmission range of buried smart objects in IoUT is still a significant issue which is still an open research problem.

\section{Low Latency and Reliable Communications}

Most of the applications of IoUT require low latency and reliable communication. For example, the underground things are deployed in the oil and gas wells to perform critical sensing tasks, such as temperature/pressure, pipe leaks and gas leaks, and therefore the data should be reliable and received at the surface with low latency. If the sensed data from even a single sensor is not received on time, it can lead to a disaster. As the performance of the IoUT is highly susceptible to the harsh underground environment, it can lead to unreliable and high latency communication. Although the requirement of low latency and reliable communication is essential for both terrestrial IoT and IoUT, it is important to note that the operating environment and regulations in underground are different than the conventional IoT networks. There is no single communication system which can meet all the requirements in the harsh underground medium. For example, the wired solutions can provide reliable and low latency underground communications, but they are not scalable, are complex, and have a high cost. On the other hand, the wireless solutions are scalable, have low complexity, and have low cost but are susceptible to the harsh underground environment. Hence, a reliable and low latency architecture is required for IoUT which can count for sensor failures and minimize the transmission delay.

\section{E. Security}

Although efforts have been made in the past to model the channel for IoUT [40], [99], unfortunately, security for IoUT 
did not get much attention from the research community. Security of IoUT includes the security of equipment and the communication protocols. Various attacks, such as node replication, signal jamming, and wormhole can be launched to destabilize the operation of the IoUT. The security breach can also exhaust the network resources by triggering false alarms and responding to false alarms. Various security issues for a cloud-based IoT are addressed in [163] where the issues of malicious attack, forward and backward security, node compromise attack, and semi-trusted cloud security are identified. Similarly, the authors in [164] provided efficient data tagging technique by using information flow control (IFC) that improves the security of the data. However, tagging of resource-constrained IoT devices is expensive. Moreover, the authors in [165] presented a secure architecture for IoT networks based on datagram transport layer security (DTLS) and host identity protocol (HIP). A detailed survey on security issues in IoT networks can be found in [166]. All of the above works are focused on security and privacy for terrestrial IoT networks that can be adapted for IoUT by adding the constraints of the underground environment. For instance, the older control and information systems for oil and gas fields are transforming into a digitized IoUT network. The security mechanisms are also needed to be updated because these systems were never designed to be globally connected. Hence, such systems can easily be targeted by the cyberattacks, leading to a disaster. One solution for the security in IoUT networks is to investigate the blockchain technology, which can significantly reduce the possibility of cyber-attacks. The distributed ledger in blockchain can be an ideal solution to provide de-centralized security.

\section{F. Scalability}

Routing overhead, higher network density, and node failures can cause scalability issue for IoUT. Moreover, the high energy consumption and limited memory of underground sensor nodes limit the scalability of the network. Additionally, the IoUT system may consist of sensor nodes developed by different vendors which can lead to interoperability issues. Several works on scalability issue exist for various types of IoT networks. For example, in [167], the authors investigated the scalability issue in cellular networks for IoT devices by using spatiotemporal stochastic modeling. Similarly, structure-aware and self-adaptive wireless sensor network setup was proposed in [168] to address the scalability issue in a tunnel. Moreover, a middleware protocol was introduced in [168] to cope with the interoperability issue. The proposed protocol in [169] connects heterogeneous IoT devices. The above works address the issue of scalability and interoperability for terrestrial IoT networks. However, the harsh underground environment should be taken into account to modify these techniques for IoUT. For example, in the case of buried smart objects, the increased path loss of wireless communication signals in soil limits the connectivity of a large-scale IoUT network. In [170], the authors investigated the problem of scalability and lifetime maximization for agricultural IoUT where a mobile sink node was used to connect the sparsely deployed nodes, and energy harvesting was used to improve the network lifetime. Recently, a large-scale IoUT was implemented in [171], implementing a single-hop star topology which was able to support up to one million devices. Besides a few of the above solutions, IoUT requires the development of self-healing and self-organizing techniques to overcome the scalability issue.

\section{G. Robustness}

Robustness is another crucial issue for IoUT which has not been addressed in the past. In an underground resourceconstrained environment where there are various challenges, such as dynamic topology, energy constraint, and sparsity of nodes, robustness is critical. In [172], the authors proposed a small world model to improve the robustness and latency of heterogeneous IoT network where the local importance of the smart object was taken into account. Robustness for terrestrial IoT networks is well-studied from different perspectives. For example, form localization aspect, detailed discussion about the robustness in terrestrail IoT can be found in [173]. The literature on robustness for underground networks is only limited to the mining application. For example, a robust mesh wireless network was proposed in [174] for underground mining which enhances both reactive and precautionary measures in safety and emergency situations. Since communication in IoUT networks suffers from an extremely harsh environment, it is challenging to develop robust communication and data gathering schemes. For example, the heterogeneous soil medium and water content of the soil limit the transmission range of electromagnetic waves. However, magnetic induction is more robust to the soil medium and water contents. Nevertheless, magnetic induction suffers from low transmission range and require perfect orientation between the coils. However, the research on using magnetic induction and its robustness for underground communications is still not mature and require further investigation.

\section{H. Hybrid Sensing}

A hybrid sensing system for IoUT integrates signals from multiple types of sensor systems for the detection and localization of an event. For example, a network of longterm underground fiber sensors can be fused with short-term ground penetrating radars for the detection and localization of an underground event. Another example of hybrid sensing network is SoilNet system proposed in [175]. In SoilNet, EMwaves based Zigbee network is used for above-ground communications and wired communication is used for underground links between the buried nodes. Similarly, hybrid EM and MIbased sensing network can be used where the EM can provide long-range down-link communication while short-range MI can be used to provide multi-hop uplink communication [132]. Hence, hybrid sensing technologies and new concepts, such as crowd-sensing can proactively detect and localize underground events, and improve the efficiency of IoUT.

\section{Software Defined Networking}

Although the research on IoUT is still in its infancy, researchers are already looking to develop networking solutions for the smart objects connected underground. Unlike 
conventional networking, software-defined networking (SDN) provide a scalable, secure, and reliable solution. Due to these advantages of SDN, it is used for underwater communications systems where the underwater sensors use in/out-band control channels to communicate with the surface station [176]. The surface station acts as an SDN controller to separate the data plane and the control plane. A similar approach can be used for IoUT, to provide an appropriate networking [177]. By employing SDN for the IoUT reduces the network complexity, improve load balancing, provide congestion control, efficiently utilize the network resources, improve the network lifetime, and reduce the latency. For example, in IoUT networks for oil and gas reservoirs monitoring, SDN can provide a global network view of the underground smart objects enabling efficient management of the network. Besides, for largescale IoUT networks in agricultural applications, SDN can provide a scalable network management solution. Moreover, data visualization tools such as multidimensional scaling [178] can be incorporated at the SDN controller to correlate the data from the smart objects. Hence with all these advantages, the SDN paradigm needs to be examined for the IoUT.

\section{J. Big Data}

IoUT is going to generate a large amount of exploration data which include data coming from various applications, such as agriculture, seismic surveying, and oil/gas fields. Hence this massive amount of data need to be organized for a proper analysis, a metric calculation, or an event correlation to make accurate decisions [179]. There has been a lot of research efforts made recently to integrate IoT and big data for conventional IoT networks. For example, Atzori et al. presented an overview of enabling technologies and potential applications of big data for IoT networks [180]. Similarly, context-aware computing and its usage for IoT was discussed in [181] and the references therein. These works motivate the use of big data analytics including descriptive, predictive and prescriptive analysis for underground applications as well. For example, in oil and gas IoUT networks, a large amount of exploration data is generated where managing such a massive amount of data is of major concern to the oil and gas industry. It is reported in [182] that the Geoscientists spent half of their time in managing and processing the collected data. Big data can be used to handle this massive amount of data and conduct various analysis such as scheduling and drilling. In short, proper data analytics tools need to be developed to support the large amount of data produced in IoUT.

\section{K. Cloud and Fog Computing}

The real-time and localized operations can be enabled for IoUT by integrating it with cloud and fog computing. Moreover, cloud/fog computing can provide various services for IoUT, such as scalability, location awareness, low latency, and mobility. In the recent past cloud computing has been used to provide maintenance for the oil and gas industries while fog computing has been used to reduce the data traffic and provide the analysis of the data at the network edge [183]. Also, analysis of a large amount of data generated in oil and gas industries is a big challenge; especially, in upstream where data-rich operations occur, e.g., real-time drilling, seismic exploration, and integrated processes. Hence, fog computing can provide localized real-time analytics of the data avoiding the communication delays and ensure a faster reaction to an event. Also, it can execute the drilling operations in a closed loop from a remote side by supervising through a cloud application. Hence, in time-critical applications by the time the data reach to the cloud for analysis, the opportunity to make a decision might be gone. Therefore, fog computing techniques should be integrated with the IoUT to support the in-time decision.

\section{Robust and Accurate Localization Methods}

Localization for IoUT enables numerous applications, such as geo-tagged sensing data, monitoring of the underground environment, and optimized fracturing. Few efforts have been made in the past to find the location of buried underground objects for MI-based IoUT. For example, in [127], a testbed was developed based on MI communication to track the underground objects. Moreover, the impact of mineral and rocks on the localization accuracy was investigated in [129]. Similarly, a semi-definite programming-based localization technique was developed in [132] for MI-based underground sensor networks. Nevertheless, the achievable accuracy for MI-based underground networks was investigated in [136]. All of these works are focused on MI-based communications where the work on EM, acoustic, and VLC do not exist. Therefore, robust and accurate three-dimensional methods need to be investigated based on each technology to enable the applications as mentioned earlier.

\section{Summary AND CONCLUSIONS}

In this article, we have surveyed various communication, networking, and localization techniques for the internet of underground things (IoUT). In section II, we have presented the literature on EM waves-based IoUT where we have briefly discussed the channel model and networking solutions. The primary applications of EM waves based IoUT are in agriculture due to their low penetration depth in the soil. In section III, acoustic waves-based solutions are presented for underground communications. Due to their low frequency, acoustic waves are mostly used for seismic exploration and down-hole communication during drilling. We have gathered the acoustic-based underground communication techniques in Table III and IV, where we have briefly stated their applications. Nevertheless, in section IV we have presented a wellknown mud pulse telemetry system which is commercially used for down-hole communication but suffers from various impediments, such as attenuation, dispersion, mud pump noise, and gas leakage. Furthermore, the most recent technology for IoUT, i.e., magnetic induction is presented in section $\mathrm{V}$ and various issues addressed in literature are stated in Table $\mathrm{V}$ and VI. Moreover, high speed wired solutions, such as coaxial cable and optical fiber for the IoUT are presented in section VI. In section VIII, visible light communicationbased IoUT are briefly discussed which did not get much 
attention of the researchers yet. Finally, we have presented various future research challenges which can be investigated by the researchers. In short, the research on communication, networking, and localization for IoUT still have a long way to go and require the attention of both academia and industry.

\section{REFERENCES}

[1] "2017 world population data sheet," https://www.prb.org/2017-worldpopulation-data-sheet/, accessed: 2019-04-23.

[2] "Smarter use of natural resources can inject 2 trillion dollars into global economy by $2050 \mathrm{UN}$," https://news.un.org/en/story/2017/03/553452smarter-use-natural-resources-can-inject-2-trillion-global-economy2050-un, accessed: 2019-04-23.

[3] A. Singh, D. Kumar, and J. Hotzel, "IoT based information and communication system for enhancing underground mines safety and productivity: Genesis, taxonomy and open issues," Ad Hoc Networks, vol. 78, pp. $115-129,2018$.

[4] L. Muduli, D. P. Mishra, and P. K. Jana, "Application of wireless sensor network for environmental monitoring in underground coal mines: A systematic review," J. of Network and Computer App., vol. 106, pp. 48 $-67,2018$.

[5] C. Zhou, N. Damiano, B. Whisner, and M. Reyes, "Industrial internet of things (IIoT) applications in underground coal mines," Min Eng., vol. 69 , pp. $50-56,2017$.

[6] J. Arps and J. Arps, "The subsurface telemetry problem-A practical solution," Soc. of Petro. Engineers, vol. 56, pp. 487 - 498, 1964.

[7] P. D. Thakur, P. Agnihotri, L. Deng, A. M. Soliman, P. Kieduppatum, and W. Fernandes, "The most common impacts of drilling dynamics and environments on log-while-drilling data: A study from Abu dhabi," in Abu Dhabi Int. Petroleum Exhibition \& Conf., 2018.

[8] R. J. Schroeder, "The present and future of fiber optic sensors for the oilfield service industry: where is there a role?" in 15th Optical Fiber Sensors Conf. Techn. Digest., May 2002, pp. 39-42 vol.1.

[9] M. Hernandez, D. W. MacNeill, M. Reeves, A. D. Kirkwood, J. P. Ruszka, R. Zaeper, S. R. Lemke et al., "High-speed wired drillstring telemetry network delivers increased safety, efficiency, reliability, and productivity to the drilling industry," in SPE Indian Oil and Gas Technical Conf. and Exhibition, 2008.

[10] J. Algeroy, J. Lovell, G. Tirado, R. Meyyappan, G. Brown, R. Greenaway, M. Carney, J. H. Meyer, J. E. Davies, and I. D. Pinzon, "Permanent monitoring: taking it to the reservoir," Oilfield Review, vol. 22 , no. 1 , pp. $34-41,2010$

[11] M. L. Oelze, W. D. OBrien, and R. G. Darmody, "Measurement of attenuation and speed of sound in soils," Soil Sci. Soc. Am. J., vol. 66 , pp. 788-796, May 2002.

[12] R. Sharma and A. Gupta, "Continuous wave acoustic method for determination of moisture content in agricultural soil," Computers and electronics in agriculture, vol. 73, no. 2, pp. 105-111, 2010.

[13] A. Singer, S. Yang, and M. Oelze, "Acoustic communications: Through soils, sands, water, and tissue," The J. of the Acoustical Soc. of America, vol. 141, no. 5, pp. 3986-3987, 2017.

[14] S. Yang, O. Baltaji, Y. M. Hashash, and A. Singer, "Soilcomm: A miniaturized through-soil wireless data transmission system," The J. of the Acoustical Soc. of America, vol. 144, no. 3, pp. 1872-1872, 2018.

[15] W. R. Gardner, R. E. Hyden, E. J. Linyaev, L. Gao, C. Robbins, and J. Moore, "Acoustic telemetry delivers more real-time downhole data in underbalanced drilling operations," in IADC/SPE Drilling Conf., 2006.

[16] S.-U. Yoon, E. Ghazanfari, L. Cheng, S. Pamukcu, and M. T. Suleiman, "Subsurface event detection and classification using wireless signal networks," Sensors, vol. 12, no. 11, pp. 14862-14886, 2012.

[17] S. Yoon, L. Cheng, E. Ghazanfari, Z. Wang, X. Zhang, S. Pamukcu, and M. T. Suleiman, "Subsurface monitoring using low frequency wireless signal networks," in IEEE Int. Conf. on Pervasive Comput. and Commun. Works., Mar. 2012, pp. 443-446.

[18] E. Ghazanfari, S. Pamukcu, S.-U. Yoon, M. T. Suleiman, and L. Cheng, "Geotechnical sensing using electromagnetic attenuation between radio transceivers," Smart Materials and Structures, vol. 21, no. 12, pp. 1-17, 2012.

[19] M. Akkas, I. Akyildiz, and R. Sokullu, "Terahertz channel modeling of underground sensor networks in oil reservoirs," in IEEE Global Telecommun. Conf., 2012, pp. 543-548.

[20] M. C. Vuran, A. Salam, R. Wong, and S. Irmak, "Internet of underground things in precision agriculture: Architecture and technology aspects," Ad Hoc Networks, vol. 81, pp. 160 - 173, 2018.
[21] Z. Sun and I. F. Akyildiz, "Underground wireless communication using magnetic induction," in IEEE Int. Conf. on Commun., Jun. 2009, pp. $1-5$.

[22] F. Miramirkhani, M. Uysal, O. Narmanlioglu, M. Abdallah, and K. Qaraqe, "Visible light channel modeling for gas pipelines," IEEE Photonics J., vol. 10, no. 2, pp. 1-10, Apr. 2018.

[23] S. Kisseleff, I. F. Akyildiz, and W. H. Gerstacker, "Survey on advances in magnetic induction based wireless underground sensor networks," IEEE Internet of Things $J$., pp. 1-18, Sep. 2018.

[24] M. Y. Aalsalem, W. Z. Khan, W. Gharibi, M. K. Khan, and Q. Arshad, "Wireless sensor networks in oil and gas industry: Recent advances, taxonomy, requirements, and open challenges," J. of Network and Computer Applications, vol. 113, pp. 87 - 97, 2018.

[25] X. Dong, M. C. Vuran, and S. Irmak, "Autonomous precision agriculture through integration of wireless underground sensor networks with center pivot irrigation systems," Ad Hoc Networks, vol. 11, no. 7, pp. 1975 - 1987, 2013.

[26] X. Yu, W. Han, and Z. Zhang, "Path loss estimation for wireless underground sensor network in agricultural application," Agricultural Research, vol. 6, no. 1, pp. 97-102, Mar 2017.

[27] F. Liedmann and C. Wietfeld, "SoMoS-A multidimensional radio field based soil moisture sensing system," in IEEE Sensors, Oct. 2017, pp. $1-3$.

[28] F. Liedmann, C. Holewa, and C. Wietfeld, "The radio field as a sensorA segmentation based soil moisture sensing approach," in IEEE Sensors Applications Symposium (SAS), Mar. 2018, pp. 1-6.

[29] S. Savazzi, U. Spagnolini, L. Goratti, D. Molteni, M. Latva-Aho, and M. Nicoli, "Ultra-wide band sensor networks in oil and gas explorations," IEEE Commun. Mag., vol. 51, no. 4, pp. 150-160, 2013.

[30] N. G. Franconi, A. P. Bunger, E. Sejdić, and M. H. Mickle, "Wireless communication in oil and gas wells," Energy Techno., vol. 2, no. 12, pp. 996-1005, 2014.

[31] M. Akkas, R. Sokullu, and A. Balci, "Wireless sensor networks in oil pipeline systems using electromagnetic waves," in 9th Int. Conf. on Electrical and Electronics Engineering, 2016, pp. 143-147.

[32] R. Karli, A. Bouchalkha, and K. Alhammadi, "Investigation of electromagnetic (EM) wave attenuation in oil pipeline," in Int. Conf. on Electrical and Comput. Techno. and Apps., (ICECTA), Nov. 2017, pp. $1-4$.

[33] J. Wait and J. Fuller, "On radio propagation through earth," IEEE Trans. on Antennas and Propag., vol. 19, no. 6, pp. 796-798, Nov. 1971.

[34] K. Sivaprasad and K. C. Stotz, "Reflection of electromagnetic pulses from a multilayered medium," IEEE Trans. on Geoscience Electronics, vol. 11, no. 3, pp. 161-164, Jul. 1973.

[35] R. J. Lytle, "Measurement of earth medium electrical characteristics: Techniques, Results, and Applications," IEEE Trans. on Geoscience Electronics, vol. 12, no. 3, pp. 81-101, Jul. 1974.

[36] R. J. Lytle and D. L. Lager, "The Yosemite experiments: HF propagation through rock," Radio Science, vol. 11, no. 4, pp. 245-252, Apr. 1976.

[37] W. Daily, "A new method for characterization of downhole antennas used in geophysical probing," Geophysical Research Letters, vol. 9, no. 5, pp. 507-509, 1982.

[38] W. Harrison, R. Mazza, L. Rubin, A. Yost et al., "Air-drilling, electromagnetic, MWD system development," in SPE/IADC Drilling Conference, 1990.

[39] Z. Zheng and S. Hu, "Research challenges involving cross-layered communication protocol design for underground WSNS," in 2008 2nd International Conference on Anti-counterfeiting, Security and Identification, Aug. 2008, pp. 120-123.

[40] A. R. Silva and M. C. Vuran, "Empirical evaluation of wireless underground-to-underground communication in wireless underground sensor networks," in Distributed Computing in Sensor Systems, B. Krishnamachari, S. Suri, W. Heinzelman, and U. Mitra, Eds., 2009, pp. 231-244.

[41] J. Schnitger and J. D. Macpherson, "Signal attenuation for electromagnetic telemetry systems," in SPE/IADC Drilling Conference and Exhibition, 2009, pp. 1-5.

[42] M. C. Vuran and I. F. Akyildiz, "Channel model and analysis for wireless underground sensor networks in soil medium," Physical Commun., vol. 3, no. 4, pp. $245-254,2010$.

[43] N. R. Peplinski, F. T. Ulaby, and M. C. Dobson, "Dielectric properties of soils in the 0.3-1.3-GHz range," IEEE Trans. Geosci. Remote Sens., vol. 33, no. 3, pp. 803-807, May 1995.

[44] M. T. Hallikainen, F. T. Ulaby, M. C. Dobson, M. A. El-rayes, and L. Wu, "Microwave dielectric behavior of wet soil-part 1: Empirical 
models and experimental observations," IEEE Trans. Geosci. Remote Sens., vol. 23, no. 1, pp. 25-34, Jan. 1985.

[45] A. R. Silva and M. C. Vuran, "Development of a testbed for wireless underground sensor networks," EURASIP J. on Wireless Commun. and Networking, vol. 2010, no. 1, pp. 62-74, Jan 2010.

[46] S. Yoon, L. Cheng, E. Ghazanfari, S. Pamukcu, and M. T. Suleiman, "A radio propagation model for wireless underground sensor networks," in IEEE Global Telecommun. Conf., Dec. 2011, pp. 1-5.

[47] R. Goyal, R. Kennedy, B. Kelsey, M. Whelan, and K. Janoyan, "Underground wireless sensor networks using 2nd generation RF transceivers," in Geotechnical Special Publication, no. 234, 2014, pp. 2619-2629.

[48] X. Yu, W. Han, P. Wu, and Z. Zhang, "Experiment of propagation characteristics based on different frequency channels of wireless underground sensor network in soil," Trans. of the Chinese Soc. for Agricultural Machinery, vol. 46, no. 4, pp. 252-260 and 218, 2015.

[49] G. Horvat, D. Vinko, and J. Vlaovic, "Impact of propagation medium on link quality for underwater and underground sensors," in Int. Convention on Information and Commun. Techno., Electronics and Microelectronics (MIPRO), May 2016, pp. 129-134.

[50] S. Jiang, S. V. Georgakopoulos, and O. Jonah, "RF power harvesting for underground sensors," in Proc. of the IEEE Int. Symp. on Antennas and Propagation, Jul. 2012, pp. 1-2.

[51] H. Koike and Y. Kamiya, "A new approach for subsurface wireless sensor networks," in Intelligent Interactive Multimedia Systems and Services 2016, G. D. Pietro, L. Gallo, R. J. Howlett, and L. C. Jain, Eds. Cham: Springer International Publishing, 2016, pp. 201-211.

[52] A. Salam and M. C. Vuran, "Impacts of soil type and moisture on the capacity of multi-carrier modulation in internet of underground things," in Int. Conf. on Computer Commun. and Networks, (ICCCN), Aug. 2016, pp. 1-9.

[53] _ - "Wireless underground channel diversity reception with multiple antennas for internet of underground things," in IEEE Int. Conf. on Commun., (ICC), May 2017, pp. 1-7.

[54] S. Suherman, A. Rambe, and A. Tanjung, "Underground radio propagation on frequency band $97 \mathrm{MHz}-130 \mathrm{MHz}$," Int. J. of Engineering and Techno., vol. 7, no. 3, pp. 722-726, 2018.

[55] A. Salam, M. C. Vuran, and S. Irmak, "Di-Sense: In situ realtime permittivity estimation and soil moisture sensing using wireless underground communications," Computer Networks, 2019.

[56] H. Zemmour, G. Baudoin, and A. Diet, "Soil effects on the underground-to-aboveground communication link in ultra wideband wireless underground sensor networks," IEEE Antennas and Wireless Propag. Lett., vol. 16, pp. 218-221, May 2017.

[57] D. Du, H. Zhang, J. Yang, and P. Yang, "Propagation characteristics of the underground-to-aboveground communication link about $2.4 \mathrm{GHz}$ and $433 \mathrm{MHz}$ radio wave: An empirical study in the pine forest of Guizhou province," in IEEE Int. Conf. on Computer and Commun., (ICCC), Dec. 2017, pp. 1041-1045.

[58] A. Salam, "An underground radio wave propagation prediction model for digital agriculture," Information, vol. 10, no. 4, 2019

[59] S. Conceicao, F. Ribeiro, R. Campos, and M. Ricardo, "A NS-3 based simulator of TCP/IP wireless underground networks," in IFIP Wireless Days (WD), Nov. 2014, pp. 1-6.

[60] S. Conceicao, C. Pendão, A. Moreira, and M. Ricardo, "Evaluation of medium access and a positioning system in wireless underground sensor networks," in Wireless Days (WD), Mar. 2016, pp. 1-6.

[61] L. T. Dung, H. T. H. Trang, S. Choi, and S. O. Hwang, "Impact of soil medium on the path connectivity of sensors in wireless underground sensor networks," in Int. Conf. on Adv. Techno. for Commun., (ATC), Oct. 2016, pp. 60-64.

[62] G. Liu, Z. Wang, and T. Jiang, "QoS-aware throughput maximization in wireless powered underground sensor networks," IEEE Trans. on Commun., vol. 64, no. 11, pp. 4776-4789, Nov. 2016.

[63] B. Yuan, H. Chen, and X. Yao, "Optimal relay placement for lifetime maximization in wireless underground sensor networks," Information Sciences, vol. 418-419, pp. 463 - 479, 2017.

[64] F. Adamo, G. Andria, F. Attivissimo, N. Giaquinto, and M. Savino, "An acoustic method for soil moisture measurement," in Proc. of the 20th IEEE Instrumentation Techno. Conf., vol. 2, May 2003, pp. 952-957.

[65] F. Adamo, G. Andria, F. Attivissimo, and N. Giaquinto, "An acoustic method for soil moisture measurement," IEEE Trans. Instrum. Meas, vol. 53, no. 4, pp. 891-898, Aug. 2004.

[66] R. Freire, M. H. M. de Abreu, R. Y. Okada, P. F. Soares, and C. R. GranhenTavares, "Sound absorption coefficient in situ: An alternative for estimating soil loss factors," Ultrasonics sonochemistry, vol. 22, pp. 100-107, 2015.
[67] J. M. Neff, P. L. Camwell et al., "Field test results of an acoustic telemetry MWD system," in SPE/IADC Drilling Conference, 2007.

[68] U. S. Khan, W. Al-Nuaimy, and F. E. A. El-Samie, "Detection of landmines and underground utilities from acoustic and gpr images with a cepstral approach," J. of Visual Commun. and Image Representation, vol. 21, no. 7, pp. $731-740,2010$.

[69] Z. Kang, Y. Yu, and C. Hou, "Study on stress and strain and characteristics of acoustic emission in the process of rock failure," in Second Int. Conf. on Mechanic Automation and Control Engineering, Jul. 2011, pp. 7737-7740.

[70] M. A. Gutierrez-Estevez, U. Krueger, K. A. Krueger, K. Manolakis, V. Jungnickel, K. Jaksch, K. Krueger, S. Mikulla, R. Giese, M. Sohmer, and M. Reich, "Acoustic broadband communications over deep drill strings using adaptive OFDM," in IEEE Wireless Commun. and Networking Conf., (WCNC), Apr. 2013, pp. 4089-4094.

[71] Z. Wei, S. Yibing, and L. Yanjun, "Design of acoustic wireless remote transmission system for logging-while-drilling data," in IEEE Int. Conf. on Electronic Measurement Instruments, Aug. 2013, pp. 53-57.

[72] T. J. Ahmad, M. Noui-Mehidi, and M. Arsalan, "Performance analysis of downhole acoustic communication in multiphase flow," in 40th Annual Conference of the IEEE Industrial Electronics Society, Oct. 2014, pp. 3909-3913.

[73] K. Pelekanakis, M. Chitre, L. S. Kumar, and Y. L. Guan, "Performance of channel coding and equalization for acoustic telemetry along drill strings," in IEEE Int. Conf. on Commun. Systems, Nov. 2014, pp. 610614.

[74] L. Sun and Y. Li, "Acoustic emission sound source localization for crack in the pipeline," in Chinese Control and Decision Conf., May 2010, pp. 4298-4301.

[75] B. Van Hieu, S. Choi, Y. U. Kim, Y. Park, and T. Jeong, "Wireless transmission of acoustic emission signals for real-time monitoring of leakage in underground pipes," KSCE J. of Civil Engineering, vol. 15, no. 5, p. 805, May 2011.

[76] D. Su, J. V. Miro, and T. Vidal-Calleja, "Modelling in-pipe acoustic signal propagation for condition assessment of multi-layer water pipelines," in IEEE 10th Conf. on Industrial Electronics and Applications (ICIEA), Jun. 2015, pp. 545-550.

[77] A. Alenezi and A. Abdi, "A comparative study of multichannel and single channel accelerometer sensors for communication in oil wells," in Int. Conf. on Commun. and Signal Processing (ICCSP), Apr. 2017, pp. $153-159$

[78] D. Ma, Y. Shi, W. Zhang, and G. Liu, "Design of acoustic transmission along drill strings for logging while drilling data based on adaptive ncofdm," AEU - Int. J. of Electronics and Commun., vol. 83, pp. 329-338, 2018.

[79] A. K. Farraj, S. Miller, and K. Qaraqe, "Channel characterization for acoustic downhole communication systems," in SPE Annual Technical Conf. and Exhibition. Society of Petroleum Engineers, 2012

[80] A. Farraj, "Acoustical communications for wireless downhole telemetry systems," Ph.D. dissertation, Texas A\&M University, 2012.

[81] L. S. Kumar, W. K. Han, Y. L. Guan, Y. H. Lee, and S. Sun, "Optimization of acoustic communication for industrial drilling," in IEEE Conf. on Info. Commun. Techno., Apr. 2013, pp. 1060-1063.

[82] Li, Zhigang, Ge, Shitong, and Fu, Zhongyao, "Design of the acoustic signal receiving unit of acoustic telemetry while drilling," MATEC Web of Conf., vol. 61, pp. 7-12, 2016.

[83] J. Gao, L. Chen, and Q. Li, "Study on acoustic wave transmission technology of measurement-while-drilling (MWD) data," in Int. Conf. on Adv. in Materials, Mechatronics and Civil Engineering (ICAMMCE 2018), 2018, pp. 1-6.

[84] R. Hutin, R. Tennent, S. Kashikar et al., "New mud pulse telemetry techniques for deepwater applications and improved real-time data capabilities," in SPE/IADC drilling conference, 2001.

[85] C. Klotz, P. R. Bond, I. Wassermann, and S. Priegnitz, "A new mud pulse telemetry system for enhanced MWD/LWD applications." in IADC/SPE Drilling Conf., Mar. 2008, pp. 1-8.

[86] D. Hahn, V. Peters, C. Rouatbi, and E. Scholz, "Reciprocating pulser for mud pulse telemetry," Aug. 2008, US Patent 7417920.

[87] F. Qu, Z. Zhang, J. Hu, J. Xu, S. Wang, and Y. Wu, "Adaptive dual-sensor noise cancellation method for continuous wave mud pulse telemetry," J. of Petroleum Sci. and Engineering, vol. 162, pp. $386-$ 393, 2018.

[88] Z. Jianhui, W. Liyan, L. Fan, and L. Yanlei, "An effective approach for the noise removal of mud pulse telemetry system," in Int. Conf. on Electronic Measurement and Instruments, Aug. 2007, pp. 1-9. 
[89] I. Wasserman, D. Hahn, D. H. Nguyen, H. Reckmann, and J. Macpherson, "Mud-pulse telemetry sees step-change improvement with oscillating shear valves," Oil and Gas J., vol. 106, no. 24, pp. 39-39, 2008.

[90] I. Wassermann and A. Kaniappan., "How high-speed telemetry affects the drilling process," J. of Petroleum Technology, Jun. 2009.

[91] H. Reckmann, "Downhole noise cancellation in mud-pulse telemetry," Aug. 2014, US Patent 8811118

[92] A. Jarrot, A. Gelman, and J. Kusuma, "Wireless digital communication technologies for drilling: Communication in the bits/s regime," IEEE Signal Process. Mag., vol. 35, no. 2, pp. 112-120, 2018.

[93] J. Harrell, A. G. Brooks, and H. S. Morsy, "Method and apparatus for mud pulse telemetry in underbalanced drilling systems," Aug. 2000, US Patent 6097310.

[94] Y. Lin, X. Kong, Y. Qiu, and Q. Yuan, "Calculation analysis of pressure wave velocity in gas and drilling mud two-phase fluid in annulus during drilling operations," Mathematical Problems in Engineering, vol. 2013, 2013

[95] R. Hutin, "Zero sum pressure drop mud telemetry modulator," Jan. 2016, US Patent 9228432.

[96] Y. Lu, J. Tang, Z. Ge, B. Xia, and Y. Liu, "Hard rock drilling technique with abrasive water jet assistance," Int. J. of Rock Mechanics and Mining Sciences, vol. 60, pp. 47-56, 2013.

[97] S. M. Mwachaka, A. Wu, and Q. Fu, "A review of mud pulse telemetry signal impairments modeling and suppression methods," J. of Petroleum Exploration and Production Techno., Jun 2018.

[98] C. M. Caffrey, J. Hakli, M. Hirvonen, I. Huhtinen, K. Nummila, and T. Lehikoinen, "Magnetically coupled wireless communication for buried environmental sensor," in Int. Conf. on Environment and Electrical Engineering, May 2013, pp. 341-345.

[99] X. Tan, Z. Sun, and I. F. Akyildiz, "Wireless underground sensor networks: Mi-based communication systems for underground applications." IEEE Antennas and Propag. Mag., vol. 57, no. 4, pp. 74-87, Aug. 2015

[100] I. F. Akyildiz and E. P. Stuntebeck, "Wireless underground sensor networks: Research challenges," Ad Hoc Networks, vol. 4, no. 6, pp. 669 - 686, Nov. 2006

[101] Z. Sun and I. F. Akyildiz, "Magnetic induction communications for wireless underground sensor networks," IEEE Trans. Antennas Propag, vol. 58, no. 7, pp. 2426-2435, Jul. 2010.

[102] L. Yan, D. Wei, M. Pan, and J. Chen, "Downhole wireless communication using magnetic induction technique," in United States National Committee of URSI National Radio Science Meeting (USNC-URSI NRSM), Jan. 2018, pp. 1-5.

[103] S. Kisseleff, I. F. Akyildiz, and W. Gerstacker, "On modulation for magnetic induction based transmission in wireless underground sensor networks," in IEEE Int. Conf. on Commun., (ICC), Jun. 2014, pp. 7176.

[104] A. Gungi, V. Vippalapalli, K. A. Unnikrishna Menon, and B. Hariharan, "Inductively powered underground wireless communication system," in Microelectronics, Electromagnetics and Telecommunications, S. C. Satapathy, N. B. Rao, S. S. Kumar, C. D. Raj, V. M. Rao, and G. V. K. Sarma, Eds., 2016, pp. 205-215.

[105] J. Ma, X. Zhang, and Q. Huang, "Near-field magnetic induction communication device for underground wireless communication networks," Science China Information Sciences, vol. 57, no. 12, pp. 1-11, 2014.

[106] J. Ma, X. Zhang, Q. Huang, L. Cheng, and M. Lu, "Experimental study on the impact of soil conductivity on underground magneto-inductive channel," IEEE Antennas Wireless Propag. Lett., vol. 14, pp. 17821785, Apr. 2015.

[107] A. R. Silva and M. Moghaddam, "Design and implementation of lowpower and mid-range magnetic-induction-based wireless underground sensor networks," IEEE Trans. Instrum. Meas., vol. 65, no. 4, pp. 821835, Apr. 2016.

[108] A. M. Zungeru, H. Ezea, and J. Katende, "Pulsed power system for wireless underground sensor networks," in Third Int. Conf. on Electrical, Electronics, Computer Engineering and their Applications (EECEA), Apr. 2016, pp. 126-132.

[109] H. Guo, Z. Sun, and C. Zhou, "Practical design and implementation of metamaterial-enhanced magnetic induction communication," IEEE Access, vol. 5, pp. 17213-17229, 2017.

[110] C. H. Martins, A. A. Alshehri, and I. F. Akyildiz, "Novel MI-based (FracBot) sensor hardware design for monitoring hydraulic fractures and oil reservoirs," in IEEE 8th Annual Ubiquitous Computing, Electronics and Mobile Commun. Conf., (UEMCON), Oct. 2017, pp. 434441.

[111] A. A. Alshehri, C. H. Martins, and I. F. Akyildiz, "Wireless FracBot (sensor) nodes: Performance evaluation of inductively coupled near field communication (NFC)," in IEEE Sensors Applications Symposium (SAS), Mar. 2018, pp. 1-6.

[112] H. Guo and Z. Sun, "Channel and energy modeling for self-contained wireless sensor networks in oil reservoirs," IEEE Trans. Wireless Commun., vol. 13, no. 4, pp. 2258-2269, Apr. 2014.

[113] M. A. Akkas, "Channel modeling of wireless sensor networks in oil," Wireless Personal Commun., vol. 95, no. 4, pp. 4337-4355, Aug. 2017.

[114] Z. Sun and B. Zhu, "Channel and energy analysis on magnetic induction-based wireless sensor networks in oil reservoirs," in IEEE Int. Conf. on Commun., (ICC), Jun. 2013, pp. 1748-1752.

[115] Z. Sun and I. F. Akyildiz, "Optimal deployment for magnetic inductionbased wireless networks in challenged environments," IEEE Trans Wireless Commun., vol. 12, no. 3, pp. 996-1005, Mar. 2013.

[116] A. Kulkarni, V. Kumar, and S. B. Dhok, "Enabling technologies for range enhancement of MI based wireless non-conventional media communication," in Int. Conf. on Computing, Commun. and Networking Techno., (ICCCNT), Jul. 2018, pp. 1-7.

[117] S. Swathi and S. Santhanam, "An efficient MI waveguide based underground wireless communication for smart irrigation," in IEEE India Council Int. Conf., (INDICON), 2018.

[118] V. Pathak, V. Kumar, and R. K. Barik, "Magnetic induction communication based transceiver coil and waveguide structure modeling for non-conventional WSNs," in Int. Conf. on Comput., Commun. and Networking Techno., (ICCCNT), Jul. 2018, pp. 1-7.

[119] S. Kisseleff, I. F. Akyildiz, and W. Gerstacker, "Interference polarization in magnetic induction based wireless underground sensor networks," in IEEE Int. Symposium on Personal, Indoor and Mobile Radio Commun., (PIMRC Workshops), Sep. 2013, pp. 71-75.

[120] S. Kisseleff, W. Gerstacker, Z. Sun, and I. F. Akyildiz, "On the throughput of wireless underground sensor networks using magneto-inductive waveguides," in IEEE Global Commun. Conf., (GLOBECOM), Dec 2013, pp. 322-328.

[121] S. Kisseleff, I. F. Akyildiz, and W. H. Gerstacker, "Digital signal transmission in magnetic induction based wireless underground sensor networks," IEEE Trans. on Commun., vol. 63, no. 6, pp. 2300-2311, Jun. 2015.

[122] S. Lin, I. F. Akyildiz, P. Wang, and Z. Sun, "Distributed cross-layer protocol design for magnetic induction communication in wireless underground sensor networks," IEEE Trans. on Wireless Commun., vol. 14, no. 7, pp. 4006-4019, Jul. 2015.

[123] S.-C. Lin, I. F. Akyildiz, P. Wang, and Z. Sun, "Optimal energythroughput efficiency for magneto-inductive underground sensor networks," in IEEE Int. Black Sea Conf. on Commun. and Networking (BlackSeaCom), May 2014, pp. 22-27.

[124] H. Guo and Z. Sun, "Full-duplex metamaterial-enabled magnetic induction networks in extreme environments," in IEEE Conf. on Computer Commun., (INFOCOM), Apr. 2018, pp. 558-566.

[125] H. Trang, L. Dung, and S. Hwang, "Connectivity analysis of underground sensors in wireless underground sensor networks," Ad Hoc Networks, vol. 71, pp. 104-116, 2018.

[126] A. Markham, N. Trigoni, S. A. Ellwood, and D. W. Macdonald, "Revealing the hidden lives of underground animals using magnetoinductive tracking," in Proc. of the 8th ACM Conf. on Embedded Networked Sensor Systems, 2010, pp. 281-294.

[127] A. Markham, N. Trigoni, D. W. Macdonald, and S. A. Ellwood, "Underground localization in 3-D using magneto-inductive tracking," IEEE Sensors J., vol. 12, no. 6, pp. 1809-1816, Jun. 2012.

[128] A. Markham and N. Trigoni, "Magneto-Inductive NEtworked Rescue System (MINERS): Taking sensor networks underground," in ACM/IEEE 11th Int. Conf. on Information Processing in Sensor Networks (IPSN), April 2012, pp. 1-11.

[129] T. E. Abrudan, O. Kypris, N. Trigoni, and A. Markham, "Impact of rocks and minerals on underground magneto-inductive communication and localization," IEEE Access, vol. 4, pp. 3999-4010, Aug. 2016.

[130] Q. Huang, X. Zhang, and J. Ma, "Underground magnetic localization method and optimization based on simulated annealing algorithm," in IEEE Int. Conf. on Ubiquitous Intelligence and Computing, 2016, pp. $168-173$.

[131] H. Huang and Y. R. Zheng, "3-D localization of wireless sensor nodes using near-field magnetic-induction communications," Phy. Commun., vol. 30, pp. 97-106, 2018.

[132] S. Lin, A. A. Alshehri, P. Wang, and I. F. Akyildiz, "Magnetic induction-based localization in randomly deployed wireless underground sensor networks," IEEE Internet of Things J., vol. 4, no. 5, pp. 1454-1465, Oct. 2017.

[133] T. Abrudan, Z. Xiao, A. Markham, and N. Trigoni, "Underground incrementally deployed magneto-inductive 3-d positioning network," 
IEEE Trans. Geosci. Remote Sens., vol. 54, no. 8, pp. 4376-4391, 2016.

[134] S. Kisseleff, X. Chen, I. F. Akyildiz, and W. Gerstacker, "Localization of a silent target node in magnetic induction based wireless underground sensor networks," in IEEE Int. Conf. on Commun., (ICC), May 2017, pp. 1-7.

[135] W. Tian and W. Yang, "Analytical model of transmission distance for magnetic induction through-the-earth communication under the beacon mode," J. of China Univ. of Mining and Techno., vol. 47, no. 6, pp. 1368-1377, 2018.

[136] N. Saeed, , M.-S. Alouini, and T. Y. Al-Naffouri, "On achievable accuracy of localization in magnetic inductionbased internet of underground things for oil and gas reservoirs," CoRR, vol. abs/1803.02442, 2019. [Online]. Available: http://arxiv.org/abs/1901.09556

[137] A. K. Paul and T. Sato, "Localization in wireless sensor networks A survey on algorithms, measurement techniques, applications and challenges," J. of Sensor and Actuator Networks, vol. 6, no. 4, pp. $1-24,2017$.

[138] N. Saeed, A. Celik, T. Y. Al-Naffouri, and M.-S. Alouini, "Underwater optical wireless communications, networking, and localization: A survey," Ad Hoc Networks, vol. 94, pp. 935-101, 2019.

[139] M. I. ul Haq and D. Kim, "Improved localization by time of arrival for internet of things in 3D," in Int. Conf. on Applied Electromagnetics and Commun., (ICECOM), Sep. 2016, pp. 1-5.

[140] A. R. Ansari, N. Saeed, M. I. U. Haq, and S. Cho, "Accurate 3D localization method for public safety applications in vehicular ad-hoc networks," IEEE Access, vol. 6, pp. 20756-20763, Apr. 2018.

[141] Z. Duan and Q. Zhou, "CRLB-weighted intersection method for target localization using AOA measurements," in IEEE Int. Conf. on Computational Intelligence and Virtual Environments for Measurement Systems and App., (CIVEMSA), Jun. 2015, pp. 1-6.

[142] A. Dersan and Y. Tanik, "Passive radar localization by time difference of arrival," in Proc. of MILCOM, vol. 2, Oct 2002, pp. 1251-1257 vol.2.

[143] N. Saeed and H. Nam, "Robust multidimensional scaling for cognitive radio network localization," IEEE Trans. Veh. Technol., vol. 64, no. 9 , pp. 4056-4062, 2015.

[144] _ "Cluster based multidimensional scaling for irregular cognitive radio networks localization," IEEE Trans. on Signal Process., vol. 64, no. 10 , pp. 2649-2659, 2016.

[145] — _ "Energy efficient localization algorithm with improved accuracy in cognitive radio networks," IEEE Commun. Lett., vol. 21, no. 9, 2017.

[146] N. Saeed, A. Celik, T. Y. Al-Naffouri, and M.-S. Alouini, "Underwater optical sensor networks localization with limited connectivity," in IEEE Int. Conf. on Acoustics, Speech and Signal Processing (ICASSP), Apr. 2018, pp. $1-5$

[147] — "Robust 3d localization of underwater optical wireless sensor networks via low rank matrix completion," in IEEE 19th Int. Works. on Signal Processing Adv. in Wireless Commun., (SPAWC), Jul. 2018, pp. $1-5$.

[148] N. Saeed, A. Celik, S. Alouini, and T. Y. Al-Naffouri, "Performance analysis of connectivity and localization in multi-hop underwater optical wireless sensor networks," IEEE Trans. on Mobile Comput., 2018.

[149] S. Kisseleff, X. Chen, I. F. Akyildiz, and W. H. Gerstacker, "Efficient charging of access limited wireless underground sensor networks," IEEE Trans. on Commun., vol. 64, no. 5, pp. 2130-2142, May 2016.

[150] A. A. Alshehri, S. Lin, and I. F. Akyildiz, "Optimal energy planning for wireless self-contained sensor networks in oil reservoirs," in IEEE Int. Conf. on Commun., (ICC), May 2017, pp. 1-7.

[151] R. Mijarez, D. Pascacio, R. Guevara, O. Pacheco, C. Tello, and J. Rodríguez, "Communication system for downhole measurement tools based on real-time SNR characterization in coaxial cable used as communication channel," Microelectronics Research, vol. 2013, no. 2, pp. 174-183, 2013.

[152] C. Baldwin, "Fiber optic sensors in the oil and gas industry: Current and future applications," in Opto-Mechanical Fiber Optic Sensors, H. Alemohammad, Ed. Butterworth-Heinemann, 2018, pp. 211 236.

[153] "IntelliServ is more than just wired drill pipe," https://www.nov.com/Segments/Wellbore Technologies/IntelliServ.aspx, accessed: 2019-05-05.

[154] T. K. Kragas, B. A. Williams, G. A. Myers et al., "The optic oil field: deployment and application of permanent in-well fiber optic sensing systems for production and reservoir monitoring," in SPE Annual Tech. Conf. and Exhibition, 2001.
[155] A. D. Kersey, "Optical fiber sensors for permanent downwell monitoring applications in the oil and gas industry," IEICE Trans. on electronics, vol. 83, no. 3, pp. 400-404, 2000.

[156] Y. Zhang, J. Ning, S. Yang, and H.-L. Cui, "Field test investigation of fiber optic seismic geophone in oilfield exploration," Proc. SPIE, vol. 6770, no. 8, pp. 6770 - 6770, 2007.

[157] X. Zhou, Q. Yu, and W. Peng, "Simultaneous measurement of downhole pressure and distributed temperature with a single fiber," Measurement Science and Techno., vol. 23, no. 8, pp. 8-16, 2012.

[158] B. Prevedel, F. Bulut, M. Bohnhoff, C. Raub, R. F. Kartal, F. Alver, and P. E. Malin, "Downhole geophysical observatories: best installation practices and a case history from Turkey," Int. J. of Earth Sci., vol. 104, no. 6, pp. 1537-1547, Sep. 2015.

[159] P. M. Nellen, P. Mauron, A. Frank, U. Sennhauser, and K. Bohnert, "Reliability of fiber bragg grating based sensors for downhole applications," Sensors and Actuators A: Physical, vol. 103, no. 3, pp. 364 $-376,2003$

[160] H. Wu, Y. Guo, L. Xiong, W. Liu, G. Li, and X. Zhou, "Optical fiber based sensing, measuring, and implementation methods for slope deformation monitoring: A review," IEEE Sensors J., pp. 1-20, Feb. 2019.

[161] Y. Li, S. Videv, M. Abdallah, K. Qaraqe, M. Uysal, and H. Haas, "Single photon avalanche diode (spad) VLC system and application to downhole monitoring," in IEEE Global Commun. Conf., (GLOBECOM), Dec. 2014, pp. 2108-2113.

[162] S. Kisseleff, B. Sackenreuter, I. F. Akyildiz, and W. Gerstacker, "On capacity of active relaying in magnetic induction based wireless underground sensor networks," in IEEE Int. Conf. on Commun., (ICC), Jun. 2015, pp. 6541-6546.

[163] R. Roman, P. Najera, and J. Lopez, "Securing the internet of things," Computer, vol. 44, no. 9, pp. 51-58, Sep. 2011.

[164] D. Evans and D. M. Eyers, "Efficient data tagging for managing privacy in the internet of things," in IEEE Int. Conf. on Green Comput. and Commun., Nov. 2012, pp. 244-248.

[165] O. Garcia-Morchon, S. L. Keoh, S. Kumar, P. Moreno-Sanchez, F. Vidal-Meca, and J. H. Ziegeldorf, "Securing the IP-based internet of things with HIP and DTLS," in Proc. of the Sixth ACM Conf. on Security and Privacy in Wireless and Mobile Networks, 2013, pp. 119124.

[166] S. Sicari, A. Rizzardi, L. Grieco, and A. Coen-Porisini, "Security, privacy and trust in internet of things: The road ahead," Computer Networks, vol. 76, pp. 146-164, 2015.

[167] M. Gharbieh, H. ElSawy, A. Bader, and M. Alouini, "Spatiotemporal stochastic modeling of iot enabled cellular networks: Scalability and stability analysis," IEEE Trans. on Commun., vol. 65, no. 8, pp. 35853600, Aug. 2017.

[168] M. Li and Y. Liu, "Underground structure monitoring with wireless sensor networks," in Proc. of the 6th Int. Conf. on Info. Processing in Sensor Networks, 2007, pp. 69-78.

[169] T. Vresk and I. Cavrak, "Architecture of an interoperable IoT platform based on microservices," in Int. Convention on Information and Commun. Techno., Electronics and Microelectronics (MIPRO), May 2016, pp. 1196-1201.

[170] J. Tooker and M. C. Vuran, "Mobile data harvesting in wireless underground sensor networks," in Annual IEEE Commun. Soc. Conf. on Sensor, Mesh and Ad Hoc Commun. and Netw., (SECON), Jun. 2012, pp. $560-568$

[171] X. Zhang, A. Andreyev, C. Zumpf, M. C. Negri, S. Guha, and M. Ghosh, "Invited paper: Thoreau: A fully-buried wireless underground sensor network in an urban environment," in Int. Con. on Commun. Sys. Netw., (COMSNETS), Jan. 2019, pp. 239-250.

[172] D. Luo, T. Qiu, N. Deonauth, and A. Zhao, "A small world model for improving robustness of heterogeneous networks," in IEEE Global Conf. on Signal and Info. Process., (GlobalSIP), Dec. 2015, pp. 849852.

[173] L. Chen, S. Thombre, K. Jarvinen, E. S. Lohan, A. Alen-Savikko, H. Leppakoski, M. Z. H. Bhuiyan, S. Bu-Pasha, G. N. Ferrara, S. Honkala, J. Lindqvist, L. Ruotsalainen, P. Korpisaari, and H. Kuusniemi, "Robustness, security and privacy in location-based services for future IoT: A survey," IEEE Access, vol. 5, pp. 8956-8977, 2017.

[174] G. A. Kennedy and P. J. Foster, "High resilience networks and microwave propagation in underground mines," in European Conf. on Wireless Techno., Sep. 2006, pp. 193-196.

[175] H. R. Bogena, J. A. Huisman, H. Meier, U. Rosenbaum, and A. Weuthen., "Hybrid wireless underground sensor networks: Quantification of signal attenuation in soil," Vadose Zone J., vol. 8, pp. 755-761, Aug. 2009. 
[176] I. F. Akyildiz, P. Wang, and S.-C. Lin, "SoftWater: Software-defined networking for next-generation underwater communication systems," Ad Hoc Networks, vol. 46, pp. 1-11, Aug. 2016.

[177] J. Puente Fernández, L. García Villalba, and T.-H. Kim, "Software defined networks in wireless sensor architectures," Entropy, vol. 20, no. 4, p. 225, 2018.

[178] N. Saeed, H. Nam, M. I. U. Haq, and D. B. Muhammad Saqib, "A survey on multidimensional scaling," ACM Comput. Surv., vol. 51, no. 3, pp. 47:1-47:25, May 2018

[179] M. S. Hajirahimova, "Opportunities and challenges big data in oil and gas industry," Institute of Information Techno. of Azerbaijan National Academy of Sci., Tech. Rep., 2017.

[180] L. Atzori, A. Iera, and G. Morabito, "The internet of things: A survey," Computer Networks, vol. 54, no. 15, pp. 2787 - 2805, 2010.

[181] C. Perera, A. Zaslavsky, P. Christen, and D. Georgakopoulos, "Context aware computing for the internet of things: A survey," IEEE Commun. Surv. Tut., vol. 16, no. 1, pp. 414-454, May 2014.

[182] M. Mohammadpoor and F. Torabi, "Big data analytics in oil and gas industry: An emerging trend," Petroleum, 2018.

[183] R. K. Perrons and A. Hems, "Cloud computing in the upstream oil \& gas industry: A proposed way forward," Energy Policy, vol. 56, pp. $732-737,2013$

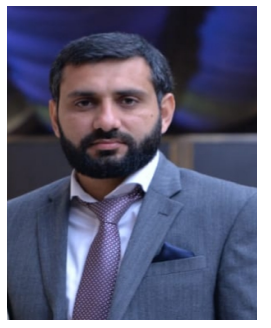

Nasir Saeed (S'14-M'16) received his Bachelors of Telecommunication degree from University of Engineering and Technology, Peshawar, Pakistan, in 2009 and received Masters degree in satellite navigation from Polito di Torino, Italy, in 2012. He received his Ph.D. degree in electronics and communication engineering from Hanyang University, Seoul, South Korea in 2015. He was an Assistant Professor at the Department of Electrical Engineering, Gandhara Institute of Science and IT, Peshawar, Pakistan from August 2015 to September 2016. Dr. Saeed worked as an assistant professor at IQRA National University, Peshawar, Pakistan from October 2017 to July 2017. He is currently a Postdoctoral Research Fellow in King Abdullah University of Science and Technology (KAUST). His current areas of interest include cognitive radio networks, underwater optical wireless communications, dimensionality reduction, and localization.

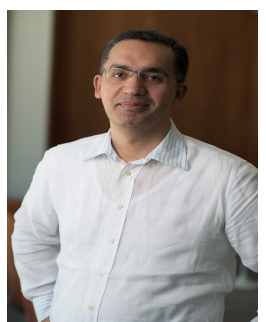

Tareq Y. Al-Naffouri (M'10-SM'18) Tareq AlNaffouri received the B.S. degrees in mathematics and electrical engineering (with first honors) from King Fahd University of Petroleum and Minerals, Dhahran, Saudi Arabia, the M.S. degree in electrical engineering from the Georgia Institute of Technology, Atlanta, in 1998, and the Ph.D. degree in electrical engineering from Stanford University, Stanford, $\mathrm{CA}$, in 2004. He was a visiting scholar at California Institute of Technology, Pasadena, CA in 2005 and summer 2006. He was a Fulbright scholar at the University of Southern California in 2008. He has held internship positions at NEC Research Labs, Tokyo, Japan, in 1998, Adaptive Systems Lab, University of California at Los Angeles in 1999, National Semiconductor, Santa Clara, CA, in 2001 and 2002, and Beceem Communications Santa Clara, CA, in 2004. He is currently an Associate Professor at the Electrical Engineering Department, King Abdullah University of Science and Technology (KAUST). His research interests lie in the areas of sparse, adaptive, and statistical signal processing and their applications, localization, machine learning, and network information theory. He has over 240 publications in journal and conference proceedings, 9 standard contributions, 14 issued patents, and 8 pending. Dr. Al-Naffouri is the recipient of the IEEE Education Society Chapter Achievement Award in 2008 and Al-Marai Award for innovative research in communication in 2009. Dr. Al-Naffouri has also been serving as an Associate Editor of Transactions on Signal Processing since August 2013.

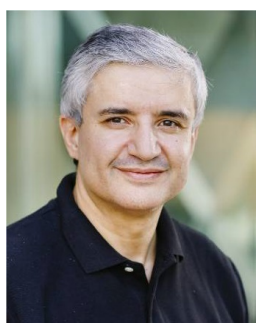

Mohamed-Slim Alouini (S'94-M'98-SM'03-F'09) was born in Tunis, Tunisia. He received the Ph.D. degree in Electrical Engineering from the California Institute of Technology (Caltech), Pasadena, CA, USA, in 1998. He served as a faculty member in the University of Minnesota, Minneapolis, MN, USA, then in the Texas A\&M University at Qatar, Education City, Doha, Qatar before joining King Abdullah University of Science and Technology (KAUST), Thuwal, Makkah Province, Saudi Arabia as a Professor of Electrical Engineering in 2009 His current research interests include the modeling, design, and performance analysis of wireless communication systems. 